\title{
X-RAY EMISSION FROM MASSIVE STARS IN CYG OB2
}

\author{
G. Rauw ${ }^{1}$, Y. Nazét ${ }^{1,10}$, N. J. Wright ${ }^{2,9}$, J. J. Drake ${ }^{2}$, M. G. Guarcello ${ }^{2}$, R. K. Prinja ${ }^{3}$, L. W. Peck ${ }^{3}$, \\ J. F. Albacete Colombo ${ }^{4}$, A. Herrero ${ }^{5}$, H. A. Kobulnicky ${ }^{6}$, S. Sciortino ${ }^{7}$, and J. S. VinK ${ }^{8}$ \\ ${ }^{1}$ Institut d'Astrophysique \& Géophysique, Université de Liège, Belgium; rauw @ astro.ulg.ac.be \\ ${ }^{2}$ Smithsonian Astrophysical Observatory, Cambridge, USA \\ ${ }^{3}$ Department of Physics and Astronomy, University College London, UK \\ ${ }^{4}$ Centro regional Zona Atlántica, Universidad Nacional del Comahue, Viedma, Argentina \\ 5 Instituto de Astrofísica de Canarias, Universidad de La Laguna, La Laguna, Spain \\ ${ }^{6}$ Department of Physics and Astronomy, University of Wyoming, Laramie, USA \\ ${ }^{7}$ INAF-Osservatorio Astronomico di Palermo, Italy \\ ${ }^{8}$ Armagh Observatory, College Hill, Armagh, UK \\ ${ }^{9}$ Centre for Astrophysics Research, University of Hertfordshire, Hatfield, UK \\ Received 2013 December 13; accepted 2014 January 28; published 2015 October 6
}

\begin{abstract}
We report on the analysis of the Chandra-ACIS data of O, B, and WR stars in the young association Cyg OB2. $\mathrm{X}$-ray spectra of $49 \mathrm{O}$-stars, $54 \mathrm{~B}$-stars, and $3 \mathrm{WR}$-stars are analyzed and for the brighter sources, the epoch dependence of the X-ray fluxes is investigated. The O-stars in Cyg OB2 follow a well-defined scaling relation between their X-ray and bolometric luminosities: $\log \frac{L_{\mathrm{X}}}{L_{\mathrm{bol}}}=-7.2 \pm 0.2$. This relation is in excellent agreement with the one previously derived for the Carina OB1 association. Except for the brightest O-star binaries, there is no general X-ray overluminosity due to colliding winds in O-star binaries. Roughly half of the known B-stars in the surveyed field are detected, but they fail to display a clear relationship between $L_{\mathrm{X}}$ and $L_{\mathrm{bol}}$. Out of the three WR stars in Cyg OB2, probably only WR 144 is itself responsible for the observed level of X-ray emission, at a very $\operatorname{low} \log \frac{L_{\mathrm{X}}}{L_{\mathrm{bol}}}=-8.8 \pm 0.2$. The X-ray emission of the other two WR-stars (WR 145 and 146) is most probably due to their O-type companion along with a moderate contribution from a wind-wind interaction zone.
\end{abstract}

Key words: open clusters and associations: individual (Cyg OB2) - stars: early-type - stars: Wolf-Rayet X-rays: stars

\section{INTRODUCTION}

Cygnus OB2 is not only interesting as a very active starforming region, but also as an association containing a wealth of massive stars. This population has been intensively studied over recent years both in terms of a general census (Comerón et al. 2002; Hanson 2003; Negueruela et al. 2008; Wright et al. 2015) as well as in terms of an extensive radial velocity survey (Kiminki et al. 2007, and Kobulnicky et al. 2012 for the most recent results). Despite its heavy extinction, Cyg OB2 is therefore an interesting place for the study of massive stars over a wide range of wavelengths, including the X-ray domain.

$\mathrm{X}$-ray emission is a well-known property of massive stars of spectral type earlier than about mid-B. For single O-stars, this emission is generally attributed to a distribution of hydrodynamic shocks produced by the so-called Line Deshadowing Instability (LDI, e.g., Feldmeier et al. 1997) in the radiativelydriven winds of these objects. Another mechanism to produce $\mathrm{X}$-ray emission from single early-type stars is the head-on collision of magnetically channeled gas in the stellar winds of massive stars that feature a strong enough magnetic field (e.g., Babel \& Montmerle 1997; ud-Doula \& Owocki 2002). Moreover, in massive binary systems, additional X-ray emission can arise from large-scale shocks associated with wind-wind interactions (e.g., Stevens et al. 1992). The shocks in magnetically confined winds and in colliding wind binaries occur at much higher Mach numbers than LDI shocks and are thus expected to produce stronger and harder X-ray emission

$\overline{{ }^{10} \text { Research Associate FRS-FNRS, Belgium. }}$ than the latter. Already in the early days of the discovery of Xray emission of early-type stars with the EINSTEIN satellite (Harnden et al. 1979), it has been found that the X-ray luminosity of O-type stars scales with their bolometric luminosity (e.g., Sciortino et al. 1990). This relationship was subsequently confirmed and refined with large samples of Otype stars observed with ROSAT (Berghöfer et al. 1997), and more recently XMM-Newton (Nazé 2009). This situation contrasts with that of Wolf-Rayet stars, for which there is no clear dependence between X-ray and bolometric luminosities (Wessolowski et al. 1996) and where some Wolf-Rayet stars remain undetected with current observatories (e.g., Oskinova et al. 2003; Gosset et al. 2005). The same holds for the lower luminosity end of massive stars, where only a subsample of the B-type stars is detected (e.g., Berghöfer et al. 1997). In the case of non-supergiant B-stars, the winds are generally thought to be too tenuous to produce strong emission via LDI shocks and alternative scenarios such as low-mass, pre-main-sequence companions and magnetic wind confinement have been suggested (Evans et al. 2011).

Despite some attempts for a theoretical explanation (Owocki \& Cohen 1999), the origin of the empirical $L_{X} / L_{\text {bol }}$ scaling relation of O-stars remained elusive for many years. From first principles, a steeper than linear relation would be expected for X-ray emission produced by LDI shocks. ${ }^{11}$ Very recently, Owocki et al. (2013) argued that the shocks in O-star winds are radiative, although the density of the winds remains in most cases sufficiently low to prevent the wind absorption from

\footnotetext{
${ }^{11} L_{\mathrm{X}}$ should scale as $L_{\mathrm{bol}}^{1.7}$ or $L_{\mathrm{bol}}^{3.4}$, respectively, for radiative or adiabatic shocks (Owocki et al. 2013).
} 
playing a significant role. Turbulence in the radiatively cooling post-shock gas, could then lead to an efficient mixing of cold and hot material. Assuming a scaling of the volume filling factor of the hot gas with some ad-hoc power $m \simeq 0.2-0.4$ of the ratio between cooling length and position in the wind, Owocki et al. (2013) were able to recover the observed $L_{\mathrm{X}} / L_{\text {bol }}$ scaling relation. Furthermore, the Owocki et al. (2013) scenario predicts a change in the behavior of the $L_{\mathrm{X}}$ versus $L_{\mathrm{bol}}$ relation at the high- and low-luminosity ends of the O-star domain, which needs to be tested observationally. Indeed, at the high-luminosity end, winds should become optically thick $^{12}$, whereas at the low-luminosity end the shocks should become adiabatic, resulting in a change of the $L_{\mathrm{X}}$ versus $L_{\text {bol }}$ relation.

The Chandra Cyg OB2 Legacy Survey offers an ideal data set for such an in-depth study of the X-ray properties of massive stars, as it provides a large and homogeneous sample of objects from early B-type, over almost all categories of $\mathrm{O}$ stars, and even several Wolf-Rayet stars.

\section{DATA ANALYSIS}

The data analyzed here are taken from the Chandra Cygnus OB2 Legacy Survey. A full description of this project and details on the data reduction are given by J. J. Drake et al. (2015, in preparation) and Wright et al. (2014). The survey consists in an overlapping $6 \times 6$ raster mosaic of $30 \mathrm{ks}$ exposures with an 8 arcmin pointing offset between adjacent fields. The sources in the central $42 \mathrm{arcmin}^{2}$ square region were typically observed four times at different off-axis angles. The survey reaches $90 \%$ completeness for $L_{X}=7 \times 10^{29} \mathrm{erg} \mathrm{s}^{-1}$ at the distance of Cyg OB2. For our variability study of O-type stars, we further include some spectra from the XMM-Newton observations discussed by Rauw (2011), Nazé et al. (2012b), and Cazorla et al. (2014).

\subsection{The Sample of Massive Stars}

Cyg OB2 has a very rich population of massive stars. The field of view of the Chandra survey contains a hundred stars classified as B-stars, 52 O-type stars and 3 Wolf-Rayet stars (Wright et al. 2015). ${ }^{13}$

\subsubsection{O-stars}

The sample of O-stars detected with ACIS spans a wide range in spectral types and luminosity classes, from O3 I to O9.5 V, although there is a clear dominance of spectral type O8 V (see Figure 1). All 52 known O-stars in the field of view are detected as X-ray emitters. The information on multiplicity of our stars was taken from the latest results of the Cygnus OB2 Radial Velocity Survey (Kiminki et al. 2007; Kobulnicky et al. 2012, 2014).

We have estimated the interstellar neutral hydrogen column density toward each source from the $E(B-V)$ color excess following the conversion formula of Bohlin et al. (1978). The color excess was evaluated from the observed $B-V$ and the

\footnotetext{
12 This transition toward optically thick winds was also found by Vink et al. (2011) in Monte Carlo radiative transfer models. These authors found a kink in the relation between $\dot{M}$ and the Eddington factor $\Gamma$ corresponding to the transition in spectral morphology from normal O/Of-type stars to Wolf-Rayet characteristics.

13 In the present study, we adopt the spectral types compiled and homogenized by Wright et al. (2015).
}

intrinsic $(B-V)_{0}$ colors as a function of spectral type according to Martins \& Plez (2006). For stars, where no $B-V$ data are available, we used the $E(J-K)$ color-excess (Negueruela et al. 2008) and the relation $E(J-K)=0.525 E(B-V)$ from Rieke \& Lebofsky (1985). As expected for observations in Cyg OB2, the interstellar absorption is quite heavy, leading to $N_{\mathrm{H}}$ values that frequently exceed $10^{22} \mathrm{~cm}^{-2}$ (see Figure 2).

\subsubsection{B-stars}

Out of 108 B-type stars that fall into the field of view covered by the survey, 54 are detected as X-ray sources. The information on spectral types and multiplicity is taken from Wright et al. (2015). Unlike the situation for O-stars, the B-star sample probably suffers from severe incompleteness and our knowledge of its multiplicity is only fragmentary.

Out of the nine known binaries in the sample, all but one are detected in X-rays. As can be seen in Figure 1, B0 stars have a higher detection rate $(75 \%)$ than B2 stars $(40 \%)$. The distribution of the interstellar column density (Figure 2) clearly indicates that absorption by the ISM has no impact on the detection or non-detection of an X-ray source associated with a B-star.

\subsubsection{Wolf-Rayet Stars}

There are three Wolf-Rayet stars that fall in the field of view of the Chandra survey: WR 144, 145, and 146. All of them are detected as X-ray sources and they are all of the WC subclass, with WR 146 being one of the few WN/WC hybrid stars (van der Hucht 2001). Two of these three Wolf-Rayet stars (WR 145 and WR 146) are actually part of binaries or higher multiplicity systems. All three stars have interstellar hydrogen column densities around $10^{22} \mathrm{~cm}^{-2}$ (see Figure 2) and are considered probable members of Cyg OB2 (Lundström and Stenholm 1984).

\subsection{Spectral fitting}

The ACIS X-ray spectra were binned in such a way as to have at least 5 counts per energy bin. They were then fitted using xspec v.12.7 (Arnaud et al. 1996). For each source, we fitted both the spectra from different observations, provided there were sufficient counts in the individual spectra ${ }^{14}$, and the total, combined ACIS spectrum. For the analysis of the global properties of the X-ray spectra (Section 3), we used only the combined ACIS spectra.

Unless stated otherwise, we used optically thin thermal plasma (apec, Smith \& Brickhouse 2001) models with solar abundances according to Anders \& Grevesse (1989). ${ }^{15}$ The majority of the spectra were fitted assuming a single plasma component. Only in those cases where the spectra are of sufficient quality and where the addition of a second plasma temperature significantly improves the fit, did we opt for a two-temperature model. The models used were thus: phab$\mathrm{s}^{*}$ phabs*apec and phabs*phabs* (apec+apec), where the phabs component indicates the absorption model. The first absorption column was fixed to the interstellar neutral hydrogen column density derived above. The second

\footnotetext{
${ }^{14}$ We require that the binned spectra must have at least four independent energy bins.

${ }^{15}$ For CCD spectra such as those investigated here, the revision of the solar composition (Asplund et al. 2009) has little impact on the results of the fit.
} 

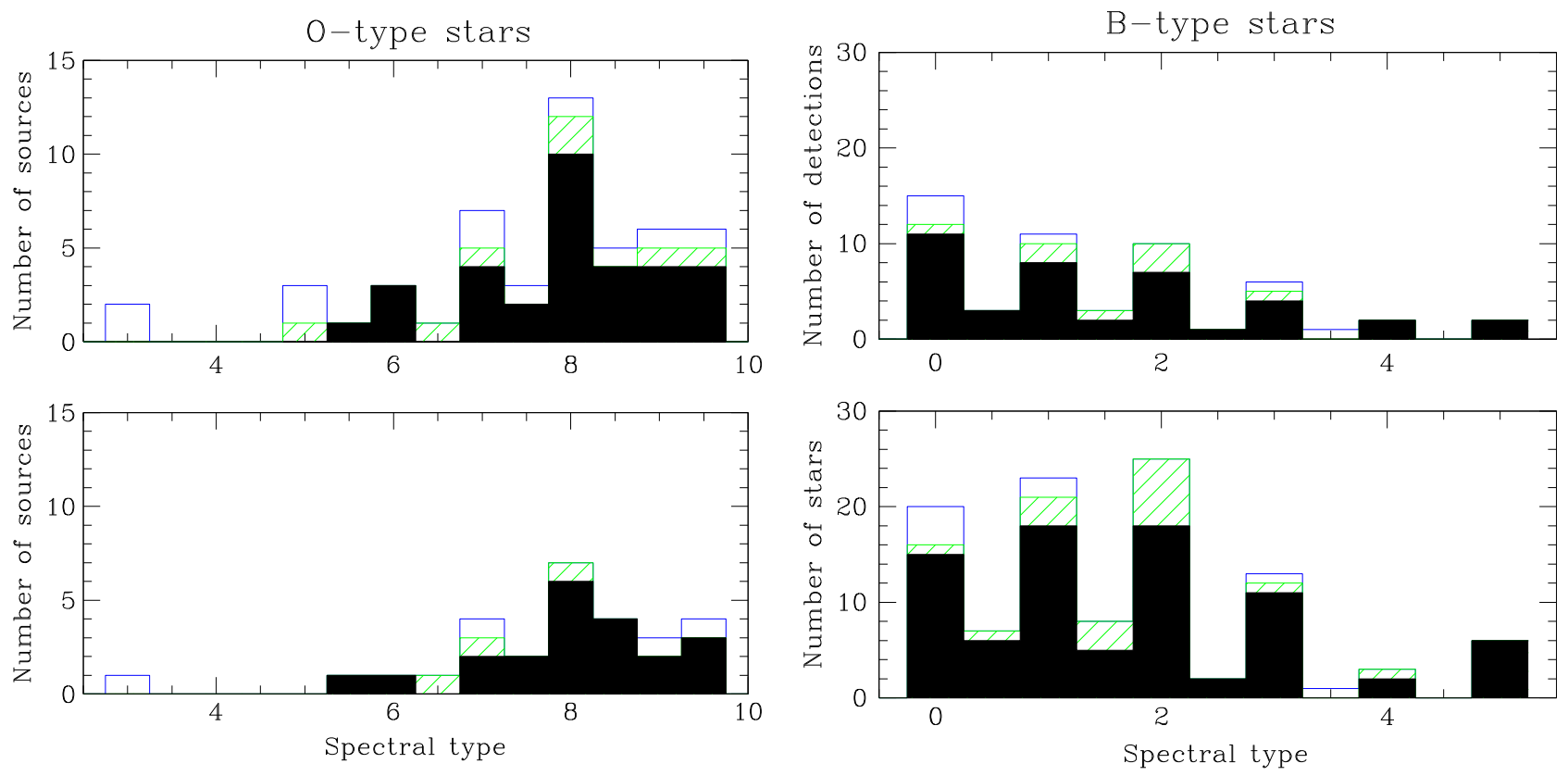

Figure 1. Left: histogram of the detected O-stars as a function of spectral types. The upper panel shows the distribution of the full sample, where the spectral type of the primary is used for binary systems. The lower panel illustrates the situation when only presumably single stars are considered. The filled, hatched and open histograms stand for main-sequence stars, giants and supergiants respectively. Right: histogram of the B-type stars as a function of spectral type. The upper panel illustrates the distribution of spectral types for those stars that are detected as counterparts of X-ray sources, while the lower panel corresponds to the full sample of known B-stars in the field of view. The various types of histograms have the same meaning as for O-stars.
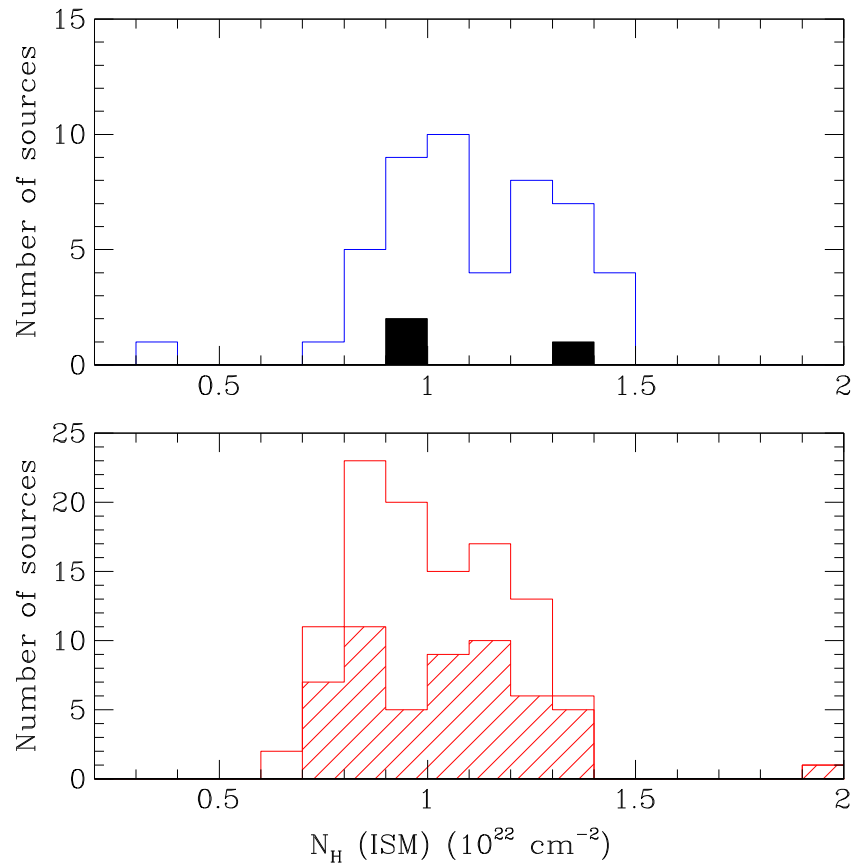

Figure 2. Top: histogram of the ISM neutral hydrogen column density toward the $\mathrm{O}$ and WR-stars in our sample. The filled histogram refers to the three Wolf-Rayet stars. Bottom: histogram of the ISM neutral hydrogen column density toward the B-type stars. The shaded histogram corresponds to the distribution for the detected objects.

absorption column is meant to represent the absorption by the stellar wind. At first sight, this might appear a crude approximation as the wind material is ionized by the photospheric radiation, while the model employed here assumes neutral material. However, this approximation impacts the spectrum only at energies below $1 \mathrm{keV}$, which are anyway absorbed by the heavy interstellar absorption in Cyg OB2. Another approximation comes from the fact that in real stellar winds, the absorbing and emitting materials are interleaved and a more sophisticated treatment of absorption is needed to fit high-resolution X-ray spectra of O-type stars (Hervé et al. 2013). Yet, for CCD spectra, such as those analyzed here, such complex models cannot be fitted and the above simple models are sufficient to provide a general description of the X-ray spectral energy distribution.

\subsection{Pile-up}

The ACIS spectra of the four brightest sources in our sample (Cyg OB2 \#8 a, \#5, \#9, and \#12) suffer from severe pile-up. XMM-Newton observations of these objects revealed orbital or long-term changes in the observed X-ray flux (Cazorla et al. 2014, and references therein).

We have evaluated the pile-up fraction of the ACIS spectra via two independent techniques. First, we applied PIMMS to the best-fit parameters inferred from the XMM-Newton spectra (De Becker et al. 2006; Linder et al. 2009; Blomme et al. 2010; Nazé et al. 2012b; Cazorla et al. 2014). Adopting the lowest fluxes observed with XMM-Newton, we estimated pile-up fractions of $60,34,18$, and $16 \%$, respectively, for on-axis Chandra observations of Cyg OB2 \#8 a, \#5, \#9, and \#12. Of course, the sources were not observed on-axis for each pointing of the Chandra campaign. For large off-axis angles, the degradation of the PSF reduces pile-up. Therefore, in a second approach, we estimated the pile-up fraction directly from the data, by looking at the maximum count rate of all the pixels within the PSF. This approach confirms that the four brightest sources are most of the time subject to pile-up fractions well above $10 \%$. The Chandra data of these sources are thus not considered further in this paper. 
The next brightest sources of our sample are CPR2002 A20, CPR2002 A11, MT91 516, Cyg OB2 \#3, and WR 146. The former and the latter two stars fall outside the field of view covered by the XMM-Newton observations (Rauw 2011) and we can only estimate the pile-up fraction from the ChandraACIS data themselves. For the two remaining stars, we proceeded in the same way as for the four brightest objects, relying on the parameters derived by Rauw (2011) for the conversion from XMM-Newton data. In this way, we estimate pile-up fractions of $3-10 \%$ for these five stars, except for ObsID 10961 where the actual data indicate a $15 \%$ pile-up fraction for MT91 516. We keep these objects in our analysis, although their spectra are probably affected by a moderate level of pile-up. As a test, we have repeated the spectral fits, using the pileup model implemented in xspec and based on the work of Davis (2001). The best-fit parameters are usually in good agreement with those obtained without pile-up corrections. The fluxes (both observed and absorption corrected) are on average 5-6\% larger than without pile-up correction. Because of some degeneracy between the plasma parameters and the pile-up model, some of the individual spectra yield larger deviations. We will thus include both solutions (with pile-up correction and without) when discussing the variability of these five sources.

All other O-stars in our sample should have pile-up fractions of less than 5\%, and we did not apply a correction in these cases.

\section{SPECTRAL ANALYSES}

\subsection{O-type Stars}

Excluding the three O-stars with heavy pile-up (Cyg OB2 \#8 a, \#5, and \#9), we are left with a sample of 49 O-type stars. The fits of the spectra of 10 objects required two plasma components with different temperatures, while the remaining spectra were well fitted with a single plasma component. The distributions of the best-fit temperatures and wind column densities are shown in Figure 3.

For single temperature model fits, there is a continuous distribution of $k T$ from about 0.1 to $1.4 \mathrm{keV}$, with a prominent peak between 0.5 and $0.6 \mathrm{keV}$. This looks quite different from the temperature distribution found for the O-stars in the Carina Nebula (Nazé et al. 2011), which is rather flat between 0.1 and $0.7 \mathrm{keV}$ and lacks objects with higher values of $k T$. However, for CCD spectra with a low number of counts, there is a wellknown degeneracy between the column density and the plasma temperature. In the present case, the fact that we have found a significant number of objects that apparently lack a wind column density in the best-fit model (see below) could bias $k T$ toward higher temperatures. To test this hypothesis, we also show in Figure 3 the distribution of $k T$ for those objects where the best-fit wind column density is different from zero. This distribution actually lacks the plasma temperatures between 0.5 and $0.6 \mathrm{keV}$ that dominate the histogram of the entire data set. This confirms that most of the higher best-fit plasma temperatures are associated with a zero wind column density. For 2-T fits, the lower temperature is generally below $0.3 \mathrm{keV}$ with a few exceptions. The second temperature spans a wide range of values, between 0.6 and $3 \mathrm{keV}$.

For 27 stars out of the 39 fitted with a single plasma component, the best-fit column of the wind absorption is found to be zero. One may thus wonder whether there are biases in our sample against the detection of a wind column. The most obvious candidates for such biases are the strong interstellar absorption toward most objects in Cyg OB2 and the low flux level of some of the sources. We have tested both hypotheses. The interstellar column has no clear impact on the detection of additional wind absorption. There are however some hints for a potential bias against such a detection for the lowest flux objects (see Figure 4).

Figure 5 shows a comparison of the observed fluxes inferred from the ACIS spectra analyzed here with the XMM-Newton EPIC-pn count rates reported by Rauw (2011) for 17 objects in common. Generally speaking, we find that the EPIC-pn count rates of most objects are larger than expected from the fluxes that we infer from the ACIS spectra. In some cases, the difference amounts to a factor two $(0.3 \mathrm{dex})$. We have also compared the fluxes obtained here with those derived by Nazé (2009) using the $2 X M M$ catalog. The fluxes of the brighter sources are in good agreement, whereas there are rather large discrepancies for the fainter objects. While some of the discrepancies could be due to variability (see below), it seems extremely unlikely that all stars would be fainter at the time of the Chandra observations.

The current status of the cross-calibration of the ChandraACIS and XMM-Newton-EPIC instruments is discussed by Schellenberger et al. (2013). These authors compare the fluxes inferred from a sample of more than 50 clusters of galaxies that are fitted with optically thin thermal plasma models, as are our stellar spectra. These authors find that ACIS yields lower flux than EPIC, mainly in the lower energy range $(0.7-2.0 \mathrm{keV})$ with typical differences of about $10 \%$. This is less than the differences found here, but one has to bear in mind that Schellenberger et al. (2013) focus on extended sources in an otherwise rather empty environment, whereas our study is concerned with point sources in a crowded environment. Therefore, the most likely reasons for the larger discrepancies in our case are source confusion in crowded regions with $X M M-N e w t o n$, due to its coarser point-spread function (PSF), and photon loss due to pile-up of the brighter sources for Chandra. We have inspected the impact of applying a wider extraction region (such as required for XMM-Newton data) to the treatment of the ACIS data. Compared to a 2.5 arcsec extraction radius (as used typically for the ACIS data), an extraction radius of 28 arcsec leads to a 50-100\% increase in the number of photons associated with a moderately bright source. This is due to contamination by a weak diffuse emission and/or a conglomerate of faint point sources. The situation is even worse for sources such as Cyg OB2 \#8c where the extraction region in the $X M M-N e w t o n$ data is affected by the wings of the PSF of the very bright Cyg OB2 \#8a. In summary, we conclude that most of the discrepancies seen on the fainter sources indeed stem from a contamination of the EPIC spectra by neighboring sources.

\subsection{The $L_{X} / L_{\text {bol }}$ Relation of $O$-stars in Cyg $O B 2$}

To investigate the relation between X-ray luminosity and bolometric luminosity, we rely on the X-ray fluxes and bolometric fluxes, which have the advantage of being independent of the distance of Cyg OB2. The X-ray fluxes were inferred from the best-fit models of the ACIS spectra. These fluxes were corrected for the interstellar absorption only, i.e., they were not corrected for additional wind absorption. Errors on the absorption corrected fluxes were estimated via the 

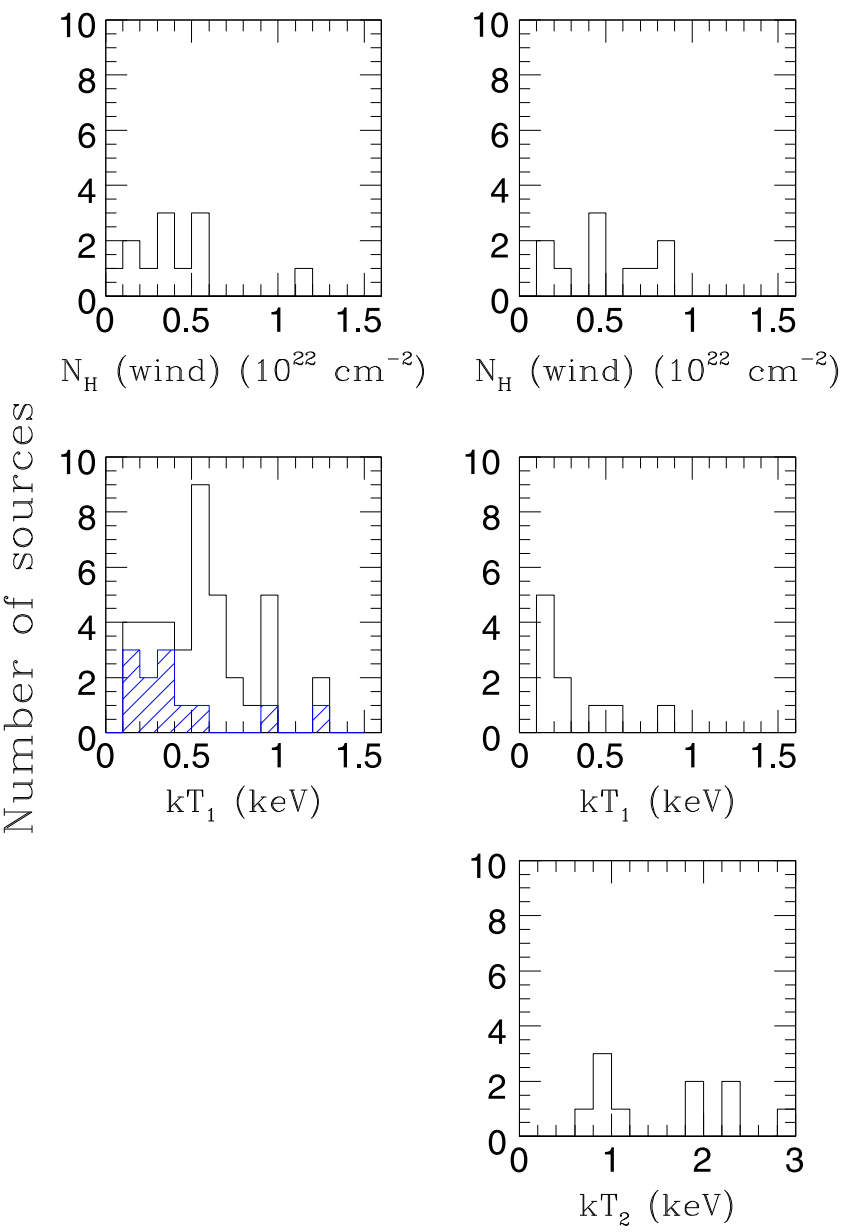

Figure 3. Histograms of the wind column densities and temperatures obtained from our fits of the spectra of 49 O-type stars in Cyg OB2. The left column corresponds to the 1-T fits (39 stars), while the right column yields the results for 2-T models (10 objects). Twenty-seven of the 39 objects with 1-T model fits have a zero wind column density. These objects are not shown in the $N_{\mathrm{H}}$ (wind) histogram. The hatched histogram in the distribution of $k T$ for 1-T fits corresponds to objects with a significant wind column density.

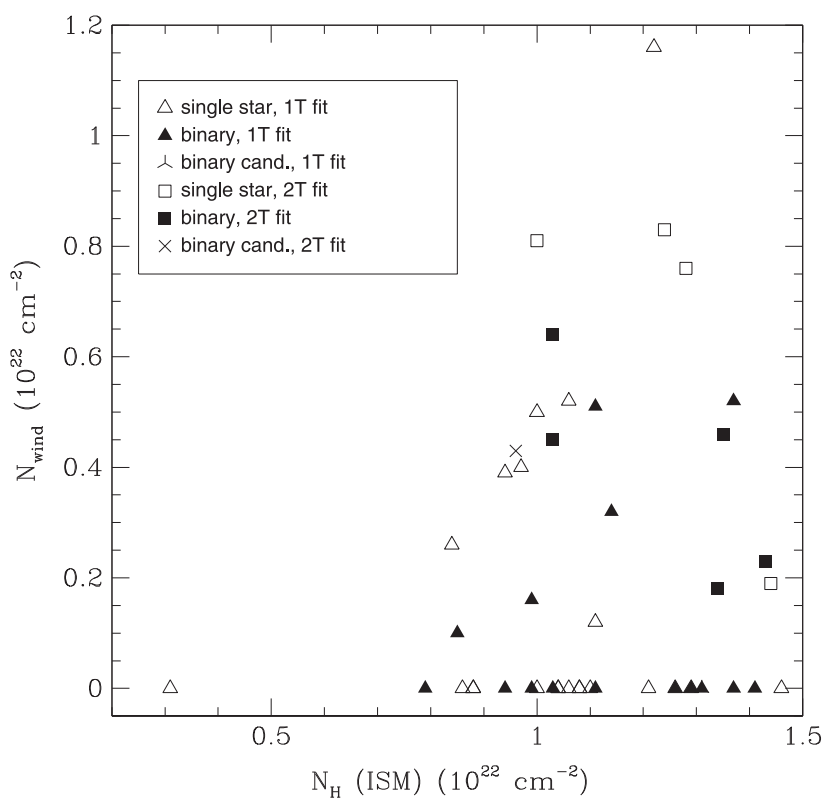

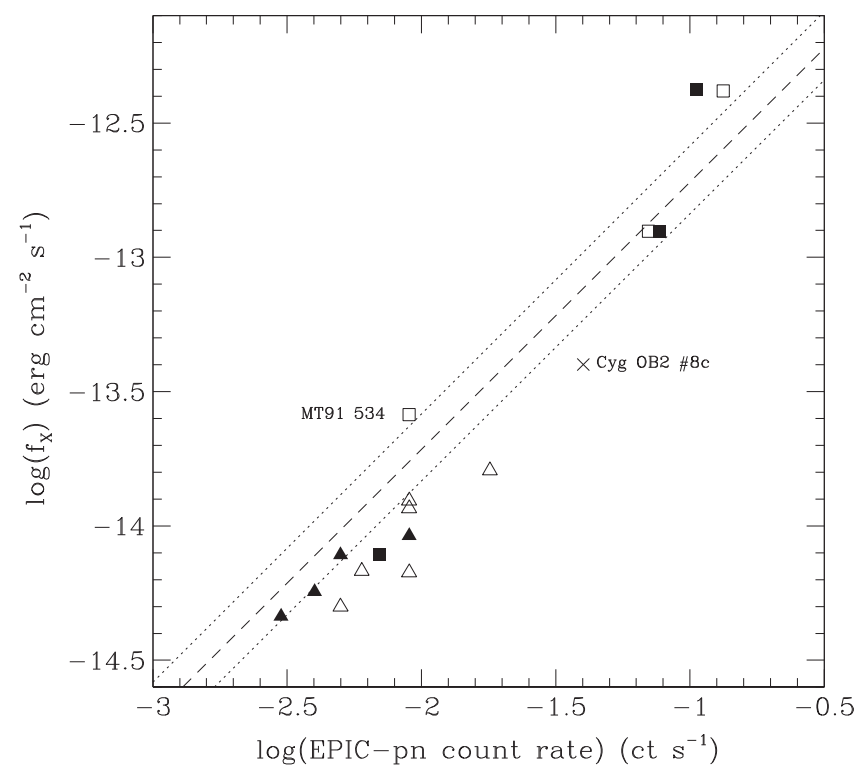

Figure 5. Comparison of the observed fluxes inferred from the ACIS spectra and the count rates of the EPIC-pn instrument aboard XMM-Newton (from Rauw 2011). The symbols have the same meaning as in Figure 4. The dashed straight line yields the conversion factor for a spectral model with the parameters that are the mean values of those inferred here $\left(N_{\mathrm{H}}(\mathrm{ISM})=\right.$ $1.07 \times 10^{22} \mathrm{~cm}^{-2}, N_{\mathrm{H}}($ wind $\left.)=0.12 \times 10^{22} \mathrm{~cm}^{-2}, \mathrm{kT}=0.56 \mathrm{keV}\right)$. The dotted lines correspond to conversion factors for models with one standard deviation about the mean spectral parameters.

cflux command in xspec. The bolometric fluxes were computed from the $V$ magnitudes, assuming a value of $R_{V}=3.1$ and adopting the bolometric corrections of Martins $\&$ Plez (2006). When no $B, V$ photometry was available, we relied on the near-IR photometry of Negueruela et al. (2008). The resulting relation between X-ray flux and bolometric flux is shown in Figure 6. This plot reveals the well-known empirical scaling relation between the bolometric and X-ray luminosities of O-type stars (see Nazé 2009 and references therein).

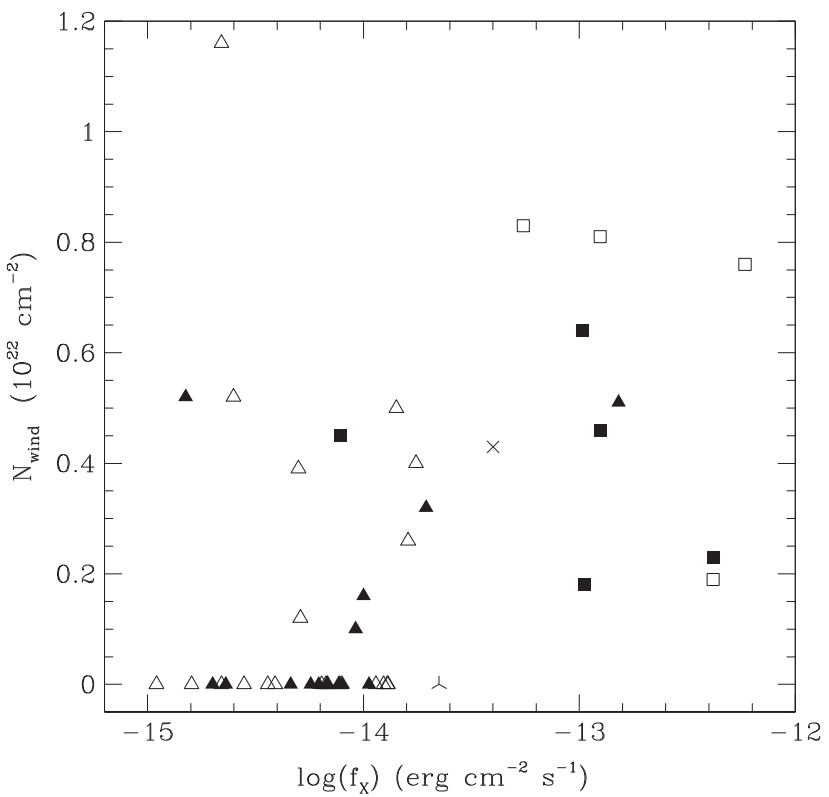

Figure 4. Left: best-fit wind column density as a function of interstellar neutral hydrogen column density. Right: best-fit wind column density as a function of observed flux in the $0.5-10 \mathrm{keV}$ domain. 

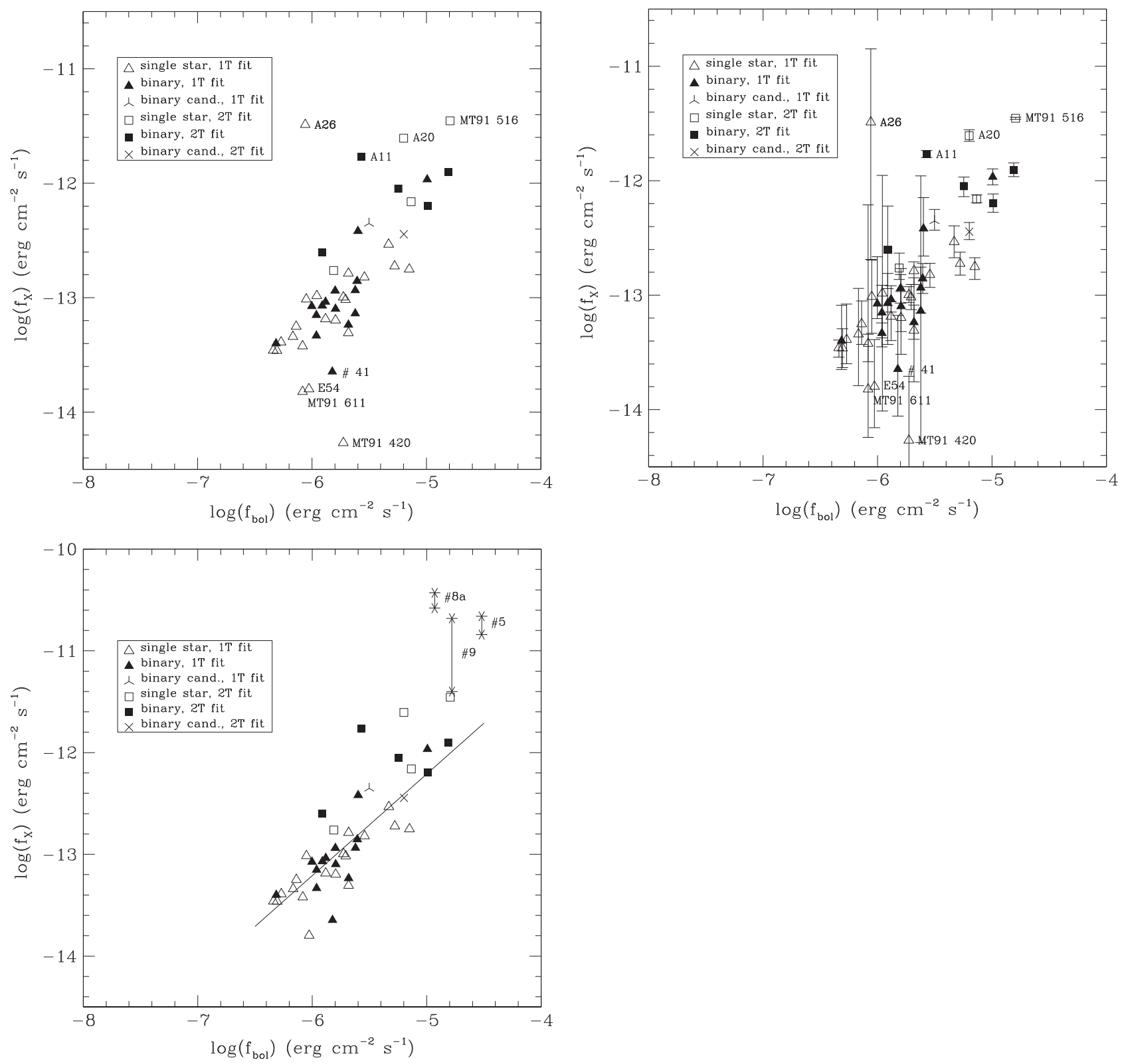

Figure 6. Top left: relation between the X-ray luminosity and the bolometric luminosity of the O-stars in Cyg OB2 as inferred from the ACIS spectra. The X-ray fluxes are evaluated in the $0.5-10 \mathrm{keV}$ domain and are corrected for the interstellar absorption. Top right: same, but with error bars derived via the cflux tool of xspec. Bottom left: same as top left, but this time restricting ourselves to results for spectra with more than 30 counts. The straight line corresponds to the scaling relation given by Equation (1). For comparison, the ranges of X-ray fluxes of the multiple systems Cyg OB2 \#5, 8 a, and 9 as measured with XMM-Newton are also shown by the asterisks.

Early-type binaries are a priori expected to be more luminous in X-rays as a result of the violent wind-wind interactions (e.g., Stevens et al. 1992). However, Figure 6 does not show a clear overluminosity of the known binary systems in Cyg OB2. ${ }^{16}$ The same conclusion was already reached by Oskinova (2005) from a sample of massive binaries observed with ROSAT, Sana et al. (2006) for O-type stars in NGC 6231, Nazé (2009) for the general sample of O-type stars observed with XMM-Newton, Nazé et al. (2011) for the O-stars in the Carina complex, and, most recently, by Nazé et al. (2013b) for the O-star population

\footnotetext{
${ }^{16}$ We note however that this statement does not apply to the three X-ray brightest $\mathrm{O}$-stars, Cyg OB2 \#5, 8 a, and 9. These three multiple systems are clearly overluminous (see bottom left panel of Figure 6), especially at phases/ epochs when their X-ray flux reaches its maximum (see also Cazorla et al. 2014).
}

of IC 2944-8 and Havlen-Moffat 1. In our sample, the strongest over-luminosities are found for CPR2002 A26, CPR2002 A20, MT91 516, and CPR2002 A11. The corresponding points are labeled in Figure 6. The X-ray flux of the first of these objects is almost certainly spurious. Indeed, the ACIS spectrum of CPR2002 A26 has a very low number of counts and the error bar on the net flux is huge (see Figure 6). Its large absorption corrected X-ray flux results from the correction of an apparently low-temperature plasma $\left(k T=0.14_{-.05}^{+.14} \mathrm{keV}\right)$ by the effect of a large interstellar column density $\left(N_{\mathrm{H}}=1.26 \times 10^{22} \mathrm{~cm}^{-2}\right)$. The position of this point should thus be taken with extreme caution. The other outliers have much higher quality spectra and are thus robust. Conversely, there are a number of underluminous stars toward the lower end of the range in bolometric luminosity explored by our sample. 
These are MT91 611, 420, and CP2012 E54 (three presumably single stars with a 1-T fit) and Cyg OB2\#41 (a binary candidate). The spectra of some of these objects are again of poor quality. We have thus built a new version of the figure, where we have discarded those data points that correspond to spectra with less than 30 counts (lower left panel of Figure 6).

We can then adjust the $L_{\mathrm{X}} / L_{\mathrm{bol}}$ relation of the O-stars in Cyg OB2. For this purpose, we exclude the five data points corresponding to spectra with less than 30 counts, as well as the three overluminous systems (CPR2002 A11, A20, and MT91 516), and we combine the data of Cyg OB2 \#22A and \#22B (see Section 4.2.1.3). Let us start with a simple scaling law between the X-ray flux and the bolometric flux (see also Appendix A). We obtain the following result:

$$
\log L_{\mathrm{X}} / L_{\mathrm{bol}}=-7.21 \pm 0.24,
$$

where all 40 data points were given equal weight in the fit. This result is in perfect agreement with the scaling relation found by Nazé et al. (2011) for the O-type stars in the Chandra Carina Complex Project. If we weight the data according to the square root of the number of counts in the spectrum, we obtain

$$
\log L_{\mathrm{X}} / L_{\mathrm{bol}}=-7.15 \pm 0.22 \text {. }
$$

Alternatively, we can also weight the data according to the cflux estimated errors on the X-ray fluxes. This time we find

$$
\log L_{\mathrm{X}} / L_{\mathrm{bol}}=-7.18 \pm 0.21 \text {. }
$$

Both relations are fully consistent with the unweighted result. Keeping the data points with less than 30 counts in the fits does not change the $\log \mathrm{L}_{\mathrm{X}} / L_{\text {bol }}$ relation, but increases its dispersion. Keeping also the overluminous systems further increases the dispersion and leads to a slight increase of $\log L_{\mathrm{X}} / L_{\mathrm{bol}}$ but still within the uncertainties of Equations (1)-(3). Albacete Colombo et al. (2008) reported $\log L_{\mathrm{X}} / L_{\mathrm{bol}}=-7 \pm 1$ for a sample of 26 OB stars in Cyg OB2. Their relation is consistent with ours, but with a much larger dispersion than obtained here.

Nazé (2009) obtained $\log L_{\mathrm{X}} / L_{\mathrm{bol}}=-6.97 \pm 0.20$ for $\mathrm{O}$ stars of Cyg OB2 in the $2 X M M$ catalog. This is $0.25 \mathrm{dex}$ brighter than what we find here. If we restrict the $2 X M M$ sample to those stars that are not known to be overluminous (either from XMM-Newton data or from the present study), we are left with 20 objects which have $\log L_{\mathrm{X}} / L_{\mathrm{bol}}=$ $-7.10 \pm 0.26$. The difference is now reduced to $0.1 \mathrm{dex}$. This remaining difference can stem from the issues discussed above and/or a slightly different treatment of the bolometric luminosity (e.g., differences in adopted spectral types).

Following the suggestion by Owocki et al. (2013), we have searched for a scaling of $L_{\mathrm{X}}$ with $\frac{\dot{M}}{v_{\infty}}$. For this purpose, we need to estimate the wind parameters $\dot{M}$ and $v_{\infty}$. We have based our evaluation of these parameters on the mass-loss rates inferred using the Vink et al. (2001) formalism and tabulated by Muijres et al. (2012), and on the assumption that $v_{\infty}=2.6 v_{\text {esc }}$ (Lamers et al. 1995) for all O-type stars. If we restrict ourselves to the presumably single stars in our sample (weighted according to the estimated errors on the fluxes), and discarding the two overluminous stars MT91 516 and CPR2002 A20, we

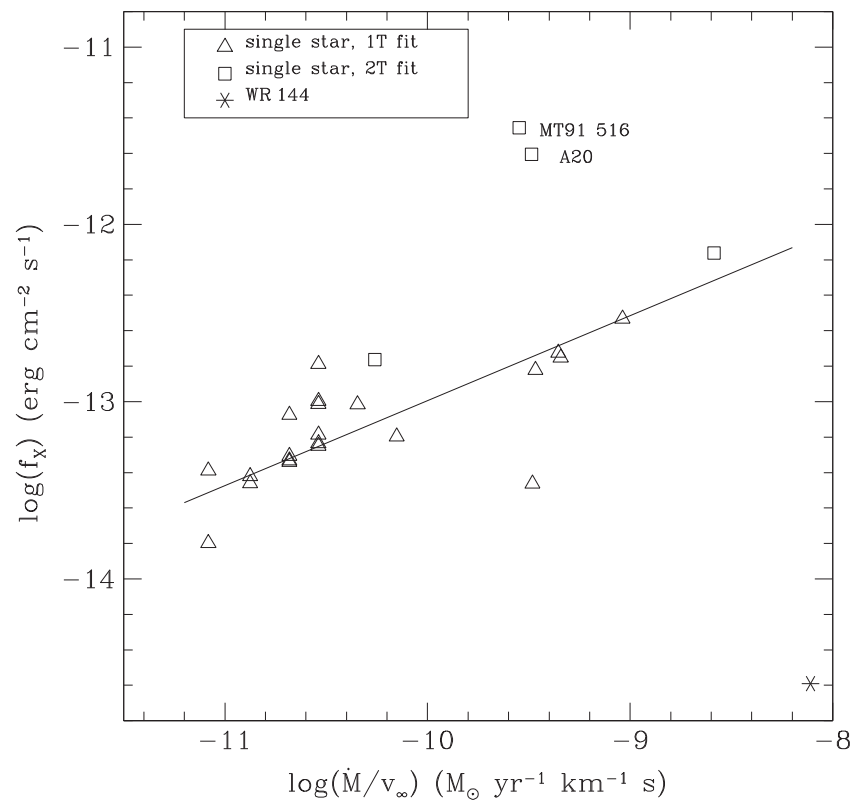

Figure 7. Logarithm of the ISM absorption corrected X-ray fluxes of presumably single O stars, and of WR 144 as a function of $\log \frac{\dot{M}}{v_{\infty}}$.

obtain

$$
\log f_{\mathrm{X}}=(0.48 \pm 0.10) \log \frac{\dot{M}}{v_{\infty}}-8.19 \pm 0.97
$$

This result is shown in Figure 7. In terms of the Owocki et al. (2013) relation, the X-ray luminosity should scale with $\left(\frac{\dot{M}}{v_{\infty}}\right)^{1-m}$ over most of the spectral range of O-type stars, where the LDI shocks that produce the $\mathrm{X}$-rays are radiative and the winds themselves are optically thin. We thus find that $m \simeq 0.52 \pm 0.10$, which is slightly larger than the upper limit of the range (0.2-0.4) proposed by Owocki et al. (2013). We note however that there is considerable scatter around the relation given by Equation (4). This could indicate that either the relation is not tight, or that the values of the wind parameters are not well enough determined with the assumptions made here.

Our current results do not show a change in the $\log \mathrm{L}_{\mathrm{X}} / L_{\mathrm{bol}}$ relation toward the higher or lower luminosity end of the O-star range, as was suggested by Owocki et al. (2013). At the lower luminosity end, we actually find that the $\log L_{\mathrm{X}} / L_{\text {bol }}$ relation of O-stars holds also for the most luminous B-stars (see Section 3.3). Concerning the high luminosity end, it must be stressed though that the Wolf-Rayet star WR 144 clearly deviates from the relation in Figure 7. Furthermore, the O-star population of Cyg OB2 does not contain $\mathrm{O} \mathrm{If}^{+}$supergiants which are likely transition objects between $\mathrm{O}$ and WR stars, and would thus be the ideal targets to search for the predicted change in the $\log L_{\mathrm{X}} / L_{\mathrm{bol}}$ relation at the highest luminosities (De Becker 2013).

As pointed out above, we find no indication of a strong $\mathrm{X}$-ray overluminosity of the known binary systems in the ACIS data. Yet it is interesting to consider this situation in a more detailed way. Wind interactions in relatively short period $\mathrm{O}+\mathrm{OB}$ systems are usually in the radiative regime where the X-ray luminosity of the wind-wind collision scales with $\dot{M} v_{\infty}^{2}$. 


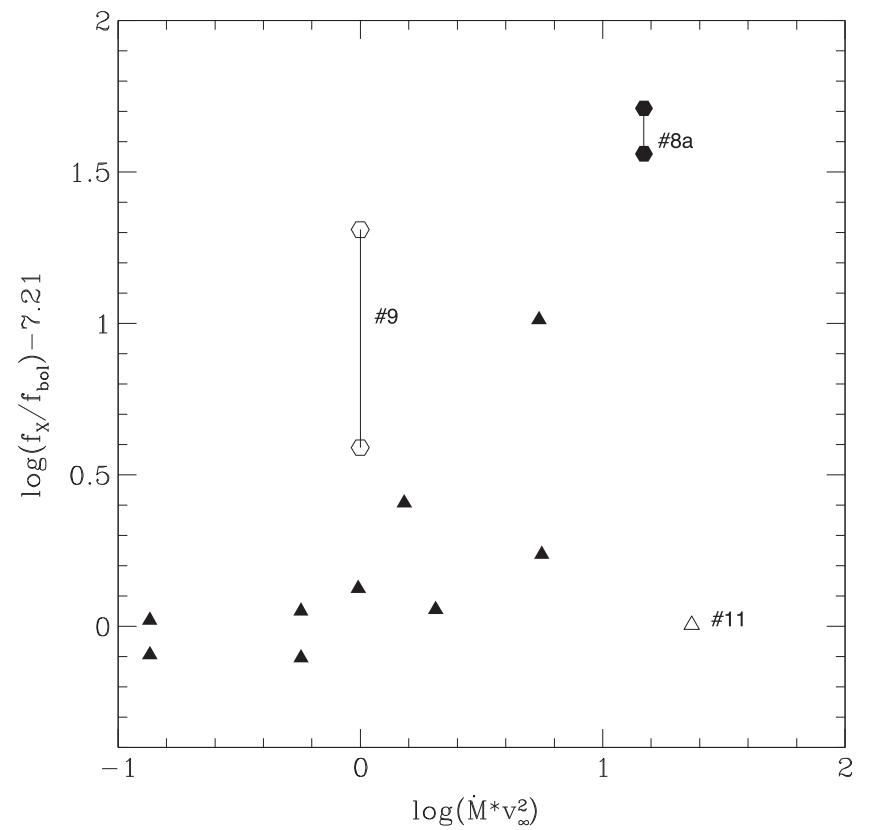

Figure 8. Overluminosity in the X-ray domain versus kinetic power of the primary wind for the known binary systems in our sample. Triangles indicate Chandra ACIS-I data, while the data for Cyg OB2 \#8a and 9 (hexagons) are taken from XMM-Newton observations. Filled symbols stand for systems with orbital periods of less than 30 days.

If we consider the known $\mathrm{O}+\mathrm{OB}$ binary systems (leaving the higher multiplicity system Cyg OB2 \#5 aside) with orbital periods shorter than 30 days, we find indeed a roughly linear increase of the X-ray overluminosity with the kinetic power of the primary star wind, although the scatter is quite substantial (see Figure 8). The two long-period systems Cyg OB2 \#9 $(P=860$ days $)$ and $\# 11$ ( $P=72$ days $)$ do not follow this trend. Nazé et al. (2012b) showed that the wind collision zone in Cyg OB2 \#9 is indeed in the adiabatic regime, where the X-ray luminosity scales with $\dot{M} /\left(v_{\infty} d\right)$, with $d$ being the orbital separation. Given its orbital period, a similar situation probably applies to Cyg OB2 \#11.

\subsection{B-Stars}

Aside from the bright blue hypergiant Cyg OB2 \#12 (Rauw 2011; Nazé et al. 2012a; Cazorla et al. 2014), which suffers from pile-up in the ACIS data, the vast majority of the B-type stars in our sample are rather faint X-ray sources. First, we have performed a spectral fit for those sources with a sufficient number of counts ${ }^{17}$ in their combined spectra (35 objects out of 51 ), using the same model as for the O-stars. There are two families in the best-fit parameters. The majority $(2 / 3)$ of the objects have a spectrum that does not require an absorption component in addition to the ISM column density. The $k T$ of these objects lies between 0.2 and $4.4 \mathrm{keV}$, with a mean of $(2.4 \pm 1.3) \mathrm{keV}$. The remaining objects apparently require additional columns in the range 0.1 to $2.2 \times 10^{22} \mathrm{~cm}^{-2}$ with a mean value of $(0.79 \pm 0.65) \times 10^{22} \mathrm{~cm}^{-2}$. The corresponding temperatures are lower (between 0.1 and $1.6 \mathrm{keV}$ ), suggesting that the additional columns are due to the above-mentioned degeneracy between temperature and column density in the fits of X-ray spectra with a low number of counts. The X-ray

\footnotetext{
${ }^{17}$ We require again a minimum of four bins in the binned spectra.
}
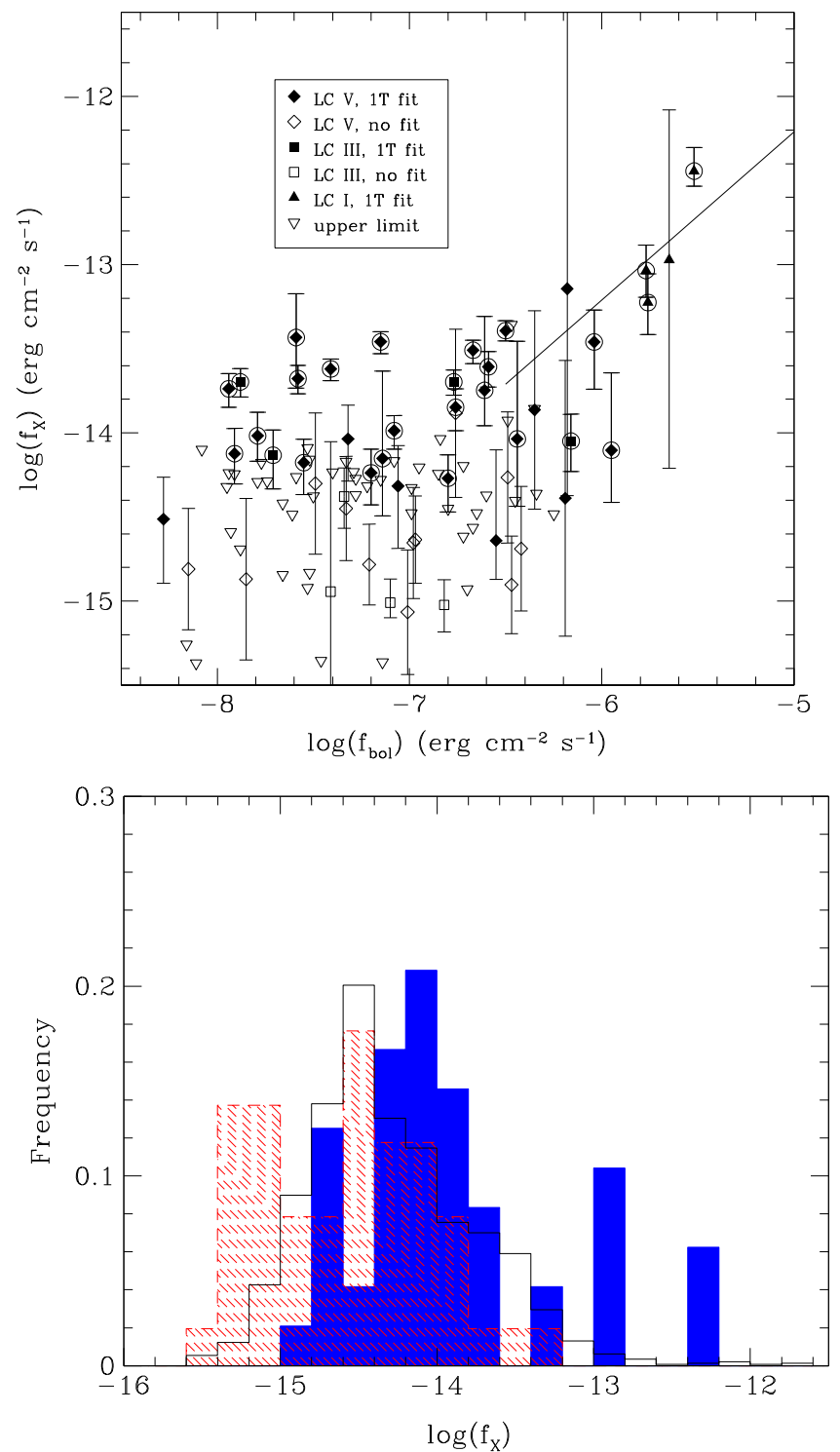

Figure 9. Top: ISM corrected X-ray fluxes of the B-type stars as a function of their bolometric fluxes. The different symbols identify the luminosity classes of the stars and indicate whether or not the fluxes were derived from a spectral fit. Symbols corresponding to objects with more than 30 counts in their spectra are encircled. The straight line indicates the best-fit relation for O-type stars. Bottom: distribution of the observed fluxes of B-type stars (hatched red histogram), O-type stars (blue filled histogram), and the full population of 1457 Cyg OB2 X-ray sources from Wright \& Drake (2009) cleaned for foreground and background sources (black empty histogram).

plasma in the B-type stars appears to be generally hotter than in the O-type stars, although there is a large dispersion in $k T$ for the B-stars. The lowest temperatures are usually found to be associated with early-type B-stars, although some late-type $\mathrm{B}$-stars also have low $k T$ and some early-type B-stars are found to have large $k T$.

We have used the above fits to derive X-ray fluxes corrected for the ISM absorption. For the 16 objects where no spectral fit could be achieved, we have derived observed and ISM absorption-corrected fluxes, assuming that their count rates are consistent with a spectrum described by a thermal plasma model with $k T=2.4 \mathrm{keV}$, absorbed by the sole ISM column. The resulting distribution of X-ray fluxes versus bolometric fluxes is shown in Figure 9. The bolometric fluxes were taken 
from Wright et al. (2015). For those known B-stars that were not detected in X-rays, we have evaluated upper limits on the $\mathrm{X}$-ray luminosities. For this purpose, 1- $\sigma$ upper limits on the number of detected counts were estimated by inserting their position into the ACIS-extract pipeline. These were then converted into photon fluxes following relation (8) of Broos et al. (2010), and into ISM absorption-corrected fluxes assuming $k T=2.4 \mathrm{keV}$ and accounting for the ISM column density. The results are shown by the downwards pointing open triangles in Figure 9.

The relation valid for O-stars that we have derived above, still holds for the brightest B-stars (early B supergiants with $\log \frac{L_{\text {bol }}}{L_{\odot}} \geqslant 4.9$, corresponding here to $\left.\log f_{\text {bol }} \geqslant-5.9\right)$. For fainter stars, there is a huge dispersion. Restricting ourselves to the brightest objects (encircled in Figure 9) would suggest a flattening of the $L_{X} / L_{\text {bol }}$ relation (see the case of NGC 6231, Sana et al. 2006). This feature as well as the generally high plasma temperatures of B-stars could reflect X-rays from magnetically confined winds (Babel \& Montmerle 1997). However, studying a sample of early-type B-stars with known magnetic fields, Oskinova et al. (2011) found that hard and strong X-ray emission does not necessarily correlate with the presence of a magnetic field. Moreover, such a flattening is not supported by the full sample. The same conclusion was reached from the data of the Chandra Carina Complex Project (Nazé et al. 2011).

The X-ray flux of the brightest non-supergiant $B$ stars reaches about $4 \times 10^{-14} \mathrm{erg} \mathrm{cm}^{-2} \mathrm{~s}^{-1}$ which, adopting a distance of $1.4 \mathrm{kpc}$, corresponds to an X-ray luminosity of about $10^{31} \mathrm{erg} \mathrm{s}^{-1}$. The latter is not inconsistent with the possibility that the X-ray emission arises from a low-mass premain sequence companion. In this context, the bottom panel of Figure 9 compares the distribution of observed fluxes of $\mathrm{O}$ and B stars with the distribution for the full set of Cyg OB2 sources from Wright \& Drake (2009) cleaned for foreground and background sources (see Wright et al. 2010). The latter should be dominated by low-mass pre-main sequence stars. Gagné et al. (2011) performed a similar exercise for the B-stars in the Chandra Carina Complex Project and found an excess of X-ray bright B-stars compared to the distribution of PMS stars. Figure 9 does not show such an excess and hence does not argue against the low-mass companion scenario.

\subsection{Wolf-Rayet Stars}

\subsubsection{WR 144}

WR 144 is a presumably single WC4 star (Sander et al. 2012, and references therein). With a net number of 5.8 counts, this star corresponds to the weakest detection of a WR star in our sample. To the best of our knowledge, this is the first X-ray detection of a presumably single WC star. Our spectrum has only two energy bins, which is not sufficient to perform a decent spectral fit. The energies of the source counts indicate a hard emission, probably as a result of a heavy circumstellar absorption. Although the data are not of sufficient quality for a spectral fit, we can use them to obtain some estimate of the $\mathrm{X}$-ray flux. For this purpose we have assumed a singletemperature model with the plasma composition as derived by Sander et al. (2012) absorbed by the ISM column and an additional wind column with the same composition as the emitting plasma. We built a grid of models with $k T$ varying between 0.3 and $3.0 \mathrm{keV}$ where the only variables correspond to the normalization parameter of the vapec component, and the wind column density. To within a factor 1.5 uncertainty, we find that the detected photons correspond to a flux (corrected for ISM absorption) of $\sim 2.55 \times 10^{-15} \mathrm{erg} \mathrm{cm}^{-2} \mathrm{~s}^{-1}$. Comparing with the bolometric flux derived from the bolometric luminosity inferred by Sander et al. (2012) yields $\log L_{\mathrm{X}} / L_{\mathrm{bol}}=-8.8 \pm 0.2$. This low value is consistent with previous results on single WC stars: Oskinova et al. (2003) reported the non-detection of the WC5 star WR 114 and argued that single WC stars are X-ray faint with $\log L_{\mathrm{X}} / L_{\text {bol }} \leqslant-8.4$ for WR 114 . Our result qualitatively and quantitatively fits into this picture.

\subsubsection{WR 145}

With a spectral type WN7/CE+O7 V((f)), WR 145 (三 MR 111) is one of a few WR stars in our Galaxy with a hybrid WN/WC spectral type. Sander et al. (2012) found that its spectrum is well fitted by a WN-type model with enhanced carbon. WR 145 is a spectroscopic binary with a period of 22.5 days showing evidence for a wind-wind interaction in the profiles of optical WR emission lines (see Muntean et al. 2009, and references therein).

As far as its observed X-ray emission is concerned, WR 145 is the brightest WR star in Cyg OB2. Its X-ray emission was already detected with EINSTEIN (Pollock 1987) and ROSAT (Pollock et al. 1995). The EINSTEIN-IPC data yield a count rate between 3 and $9 \times 10^{-3} \mathrm{cts} \mathrm{s}^{-1}$, although the differences between the different pointings are not statistically significant (Pollock 1987). ROSAT observed WR 145 twice, once during the All-Sky Survey (PSPC-C count rate of $(7.4 \pm 3.5) \times 10^{-3}$ cts s$^{-1}$ ) and once during a pointed observation (PSPC-B count rate of $(2.8 \pm 2.5) \times 10^{-3}$ cts s$^{-1}$, Pollock et al. 1995). In the Chandra survey, the star was only observed once at orbital phase 0.34 according to the ephemerides of Muntean et al. (2009). Our data thus correspond to an orbital phase shortly after quadrature with the O-star companion being closer to us than the WR star. We have fitted the spectrum with a model ${ }^{18}$ of the kind phabs ${ }^{*}$ vphabs ${ }^{*}$ vapec (see Figure 10), where the ISM column density was set to $9.7 \times 10^{21} \mathrm{~cm}^{-2}$ and the non-solar abundances were adopted, both for the emitting plasma (vapec) and the circumstellar absorption (vphabs), from Sander et al. (2012). Abundances (with respect to hydrogen) of $\mathrm{He}, \mathrm{C}$, and $\mathrm{O}$ were frozen at 1000 times solar. The $\mathrm{N}$ abundance was set to 0.001 times solar and all other elements were set at 706 times solar. A good fit $\left(\chi_{\nu}^{2}=1.08\right.$ for 110 degrees of freedom $($ dof $\left.)\right)$ is achieved with a single temperature model with $N_{\text {wind }}=\left(3.4_{-.9}^{+.6}\right) \times 10^{19} \mathrm{~cm}^{-2}$ and $k T=1.59_{-.17}^{+.38} \mathrm{keV}$. The comparatively low value of $N_{\text {wind }}$ indicates that the bulk of the X-ray emission probably arises from either the wind-wind interaction zone or the $\mathrm{O} 7 \mathrm{~V}$ companion. In this case, and given the orbital phase of our observation, one could argue that the circumstellar column toward the hot plasma might have a roughly solar abundance. In fact, adopting solar abundances for the wind column yields an equal quality fit, but this time with $N_{\text {wind }}=\left(2.82_{-.71}^{+.52}\right) \times 10^{22} \mathrm{~cm}^{-2}$. We have further tested a model with solar composition also for the emitting plasma. The

\footnotetext{
18 Zhekov (2012) analyzed the same spectrum along with several archival $X M M-N e w t o n$ spectra and concluded that a 2-T plasma model was necessary to represent the data $\left(k T_{1}=0.99, k T_{2}=4.8 \mathrm{keV}\right)$. Our fits do not require a second plasma component.
} 

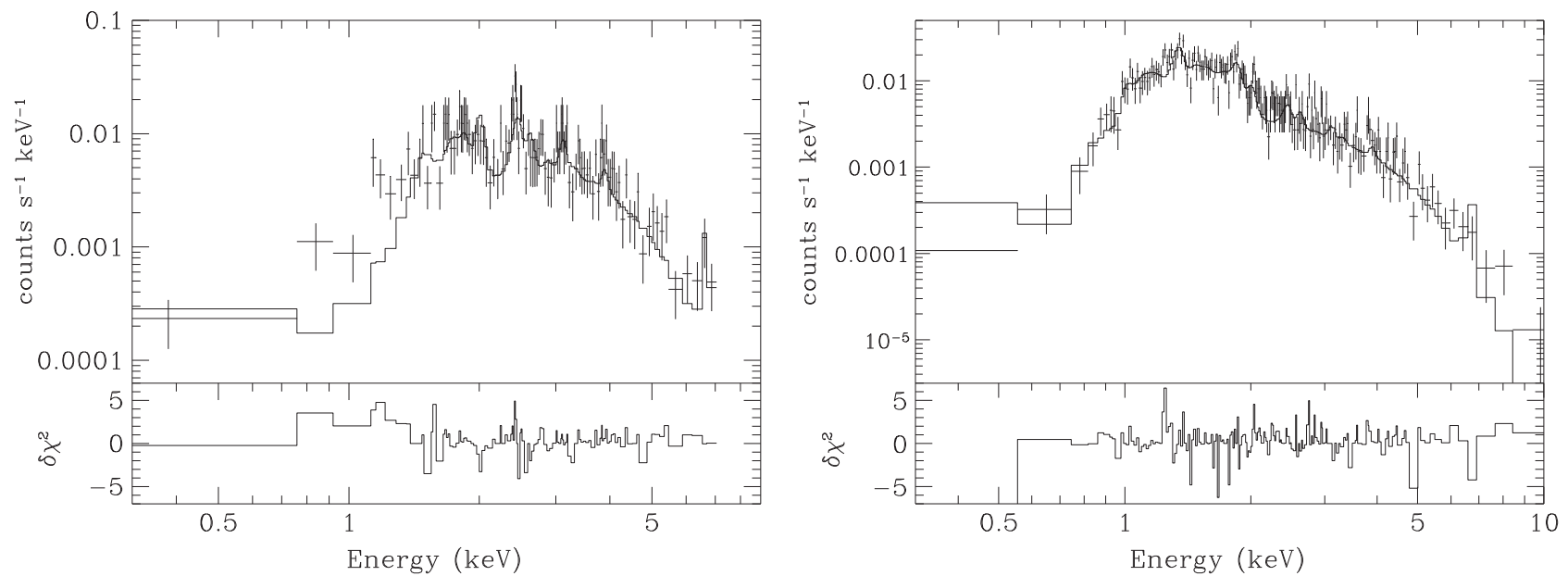

Figure 10. ACIS-I spectra of WR 145 (left) and WR 146 (right), along with their best fit assuming a non-solar composition for WR 145 and solar abundances for WR 146.

fit is actually marginally better $\left(\chi_{\nu}^{2}=1.05\right.$ for 110 dof $)$, and the parameters are $N_{\text {wind }}=\left(2.68_{-.73}^{+.53}\right) \times 10^{22} \mathrm{~cm}^{-2}$ and $k T=1.60_{-.19}^{+.43} \mathrm{keV}$. Whatever the adopted abundances, the observed and ISM corrected $\mathrm{X}$-ray fluxes are equal to $5.3 \times 10^{-13}$ and $6.5 \times 10^{-13} \mathrm{erg} \mathrm{cm}^{-2} \mathrm{~s}^{-1}$, respectively. The dereddened flux is a factor 2.5 larger than what we would expect from the sole $\mathrm{O} 7 \mathrm{~V}((\mathrm{f}))$ companion, based on our $\log \mathrm{L}_{\mathrm{X}} / \mathrm{L}_{\text {bol }}$ relation for O-type stars. Therefore, it seems probable that most of the X-ray emission of WR 145 arises from the O-star companion along with a contribution from the wind-wind interaction.

Our variability test reveals a clear intra-pointing variability of the X-ray emission of WR 145 (Kolmogorov-Smirnov statistics of $6 \times 10^{-7}$ ) during the single observation. Zhekov (2012) reported some small differences between the absorption-corrected fluxes of the various XMM-Newton and Chandra observations. We have folded our best-fit (solar composition) model through the response matrices of the EINSTEIN-IPC and ROSAT-PSPC-C instruments. The predicted count rates are $(6.7 \pm 1.1) \times 10^{-3} \mathrm{cts} \mathrm{s}^{-1}$ for the IPC, and $(1.9 \pm 1.5) \times 10^{-3}$ cts s$^{-1}$ for the PSPC-C. The agreement with the EINSTEIN data is reasonable, while the ROSAT All Sky Survey observations yields a much larger count rate than predicted here. However, given the large uncertainties (mainly due to the short integration time of the RASS on this source), this difference is not highly significant. The older X-ray data are thus not helpful to clarify the issue of variability of the X-ray flux of WR 145.

\subsubsection{WR 146}

WR 146 is a visual binary system consisting of a WC6 star with an O8 I-IIf companion (Lépine et al. 2001) at a separation of $0.168 \mathrm{arcsec}$ (Niemela et al. 1998). Radio observations reveal a thermal component associated with the WC5 star, along with an elongated non-thermal component (Dougherty et al. 1996). Using high-resolution HST images and the radio maps of Dougherty et al. (1996); Niemela et al. (1998) demonstrated that the non-thermal radio emission arises in between the two components, thereby establishing this emission as a result of the wind-wind interaction. However, the system might actually be more complex. Indeed, Setia Gunawan et al. (2000) found variations in the radio light curve of WR 146 on different timescales (decades, $3.38 \mathrm{yr}$ and weeks), and interpreted the $3.38 \mathrm{yr}$ periodicity as evidence for the presence of a third component orbiting the O8 star. A similar conclusion was reached by Dougherty et al. (2000) based on the level of radio emission, and the derived mass-loss rate, of the 08 star. These authors suggested that the latter might actually consist of an O8 star orbited by another WC star.

X-ray emission from WR 146 was previously reported with EINSTEIN (IPC count rate $6_{-.4}^{+5} \times 10^{-3} \mathrm{cts} \mathrm{s}^{-1}$, Pollock 1987) and ROSAT (PSPC-C count rate $(3.5 \pm 1.9) \times 10^{-3} \mathrm{cts} \mathrm{s}^{-1}$, Pollock et al. 1995). WR 146 was observed three times with Chandra, once in March 2007 (JD 2454177.31) and twice within one day (JD 2455258.18 and 2455258.53) in the course of the survey in 2010 March. As a first step, we have again adopted non-solar abundances from Sander et al. (2012), for both the emitting and absorbing gas. In this case, abundances (with respect to hydrogen) of $\mathrm{He}, \mathrm{C}, \mathrm{N}$, and $\mathrm{O}$ were set to 897, $1000,0.001$, and 1000 times solar. All other elements were set at 706 times solar. The spectra require a $2 \mathrm{~T}$-plasma model to achieve a good quality fit. As the model parameters of the three observations agree within the error bars, we conclude that there is no strong evidence for a time-dependence of the X-ray emission of WR 146 (see below). We thus focus on the spectrum obtained from the combination of all available ACIS data.

In all our models, the ISM column density was set to $1.32 \times 10^{22} \mathrm{~cm}^{-2}$. For the non-solar abundance model (phabs ${ }^{*}$ vphabs* vapec (2T)), the best fit $\left(\chi_{\nu}^{2}=1.32\right.$ for $176 \mathrm{dof})$ is achieved with $N_{\text {wind }}=\left(6.2_{-1.7}^{+2.1}\right) \times 10^{18} \mathrm{~cm}^{-2}$, $k T_{1}=0.36_{-.08}^{+.09}$, and $k T_{2}=2.10_{-.22}^{+.25} \mathrm{keV}$. As for WR 145, the low value of $N_{\text {wind }}$ indicates that the bulk of the X-ray emission probably arises from either the wind-wind interaction zone or the $\mathrm{O} 8$ companion. We have thus repeated the fitting process adopting solar abundances for both the emitting and absorbing gas (see Figure 10). As for WR 145, this model provides a somewhat better adjustment $\left(\chi_{\nu}^{2}=1.23\right.$ for $\left.176 \mathrm{dof}\right)$, and the parameters are $N_{\text {wind }}=\left(0.46_{-.17}^{+.20}\right) \times 10^{22} \mathrm{~cm}^{-2}, k T_{1}=0.36_{-.08}^{+.11}$, 
and $k T_{2}=2.1_{-3}^{+.4} \mathrm{keV}$. The observed and ISM-corrected X-ray fluxes are equal to $3.0 \times 10^{-13}$ and $13.6 \times 10^{-13} \mathrm{erg} \mathrm{cm}^{-2} \mathrm{~s}^{-1}$, respectively. Adopting an O8 I classification for the companion, we find that the dereddened flux agrees extremely well with the level expected from the sole O-star via our $\log L_{\mathrm{X}} / L_{\mathrm{bol}}$ relation. It thus seems quite likely that part of the $\mathrm{X}$-ray emission of WR 146 arises from the O-star companion. To further investigate the temporal dependence, we have folded our bestfit (solar composition) model through the response matrices of the EINSTEIN-IPC and ROSAT-PSPC-C instruments. The predicted count rates are $(6.7 \pm 1.1) \times 10^{-3} \mathrm{cts} \mathrm{s}^{-1}$ for the IPC and $(7.5 \pm 3.1) \times 10^{-3} \mathrm{cts} \mathrm{s}^{-1}$ for the PSPC-C. The agreement with the EINSTEIN data is very good, while the ROSAT All Sky Survey observations yield a count rate a factor two lower than predicted here. Given the uncertainties (mainly reflecting the short integration time of the RASS on this source), this difference is however not highly significant. We thus conclude that there is currently no firm evidence for long-term variations in the X-ray flux of WR 146.

\section{INTER-POINTING X-RAY VARIABILITY}

In single massive stars that emit $\mathrm{X}$-rays through the LDI mechanism, a large number of pockets of shock-heated X-ray plasma are expected to be scattered throughout the wind volume. The resulting X-ray emission is usually not seen to vary (Nazé et al. 2013a). However, considerable variability can arise either as a result of rotational modulation in single stars with magnetically confined winds (Babel \& Montmerle 1997), or in massive binary systems that host a wind interaction zone (Pittard \& Stevens 1997). The tiling strategy employed for the survey implies that most stars are observed typically four times over a six-week period. In addition, the combination of the legacy survey with older data (Wright \& Drake 2009) allows us to check for long-term variability. We can do this using either the exposure-corrected count rates or the fluxes inferred from spectral fits.

\subsection{Count Rates}

As a first step toward a quantification of the inter-pointing $\mathrm{X}$-ray variability of massive stars in Cyg OB2, we consider the photon fluxes corrected for averaged observatory response over the $[0.5,8] \mathrm{keV}$ energy band (see Equation (8) of Broos et al. 2010). For each source, we have performed a $\chi^{2}$ variability test on the count rates recorded during the various observations. Out of the 108 detected sources (51 O-stars, 54 B-stars, 3 WR stars), 23 are found to be variable at the $1 \%$ significance level. These 23 objects include the brightest stars (Cyg OB2 \#5, 8 a, 9) which suffer from pile-up in the ACIS observations. The photometry of these objects could thus also be affected and we leave them aside in the following. The results are given in Table 1 . We further identify three stars which show short-term variability (during a single observation) at a significance level of $\leqslant 1 \%$ according to a KolmogorovSmirnov test.

\subsection{Fluxes}

Based on our spectral fits, we have also investigated the variability of the X-ray flux of the brightest sources that were observed several times. We focus on the fluxes as they are generally much better constrained than other spectral model parameters, such as plasma temperatures and wind column
Table 1

Summary of the Variability Study Based on the Photon Fluxes Corrected for the Average Response

\begin{tabular}{|c|c|c|c|c|}
\hline \multirow{2}{*}{ Star } & \multirow{2}{*}{ Spectral Type } & \multicolumn{3}{|c|}{ Variability } \\
\hline & & $\begin{array}{l}\text { Inter- } \\
\text { epoch }\end{array}$ & $\begin{array}{c}\text { Intra- } \\
\text { pointing }\end{array}$ & ObsID \\
\hline \multicolumn{5}{|c|}{ O-stars } \\
\hline CPR2002 A15 & O7 I & $\mathrm{N}$ & $\mathrm{Y}$ & 12099 \\
\hline Cyg OB2 \#3 & O6 IV+O9 III & $\mathrm{Y}$ & $\mathrm{N}$ & $\ldots$ \\
\hline Cyg OB2 \#4 & O7 III & Y & $\mathrm{N}$ & $\cdots$ \\
\hline Cyg OB2 \#15 & $\mathrm{O} 8 \mathrm{~V}+\mathrm{B}$ & $\mathrm{Y}$ & $\mathrm{N}$ & $\cdots$ \\
\hline CPR2002 A11 & $\mathrm{O} 7.5 \mathrm{II}+\mathrm{OB}$ & $\mathrm{Y}$ & $\mathrm{N}$ & $\cdots$ \\
\hline Cyg OB2 \#22 & $\mathrm{O} 3 \mathrm{If}+\mathrm{O} 6 \mathrm{~V}$ & $\mathrm{Y}$ & $\mathrm{N}$ & $\cdots$ \\
\hline MT91 421 & O9 V+B9 V-A0 V & Y & $\mathrm{N}$ & $\ldots$ \\
\hline Cyg OB2 \#7 & $\mathrm{O} 3$ If & $\mathrm{Y}$ & $\mathrm{N}$ & $\ldots$ \\
\hline MT91 516 & $05.5 \mathrm{~V}$ & Y & $\mathrm{N}$ & $\ldots$ \\
\hline MT91 534 & $07.5 \mathrm{~V}$ & Y & $\mathrm{Y}$ & 10960 \\
\hline Cyg OB2 \#11 & $\mathrm{O} 5 \mathrm{If}+\mathrm{B} 0 \mathrm{~V}$ & $\mathrm{Y}$ & $\mathrm{N}$ & $\ldots$ \\
\hline Cyg OB2 \#75 & O9 V & $\mathrm{Y}$ & $\mathrm{N}$ & $\cdots$ \\
\hline Cyg OB2 \#73 & O8 III+O8 III & $\mathrm{Y}$ & $\mathrm{N}$ & $\ldots$ \\
\hline MT91 771 & $\mathrm{O} 7 \mathrm{~V}+\mathrm{O} 9 \mathrm{~V}$ & $\mathrm{Y}$ & $\mathrm{N}$ & $\cdots$ \\
\hline \multicolumn{5}{|c|}{ B-stars } \\
\hline MT91 103 & $\mathrm{~B} 1 \mathrm{~V}+\mathrm{B} 2 \mathrm{~V}$ & Y & $\cdots$ & $\ldots$ \\
\hline MT91 213 & B0 V & $\mathrm{Y}$ & $\cdots$ & $\cdots$ \\
\hline MT91 336 & B3 III & Y & $\ldots$ & $\ldots$ \\
\hline MT91 620 & B0 V & $\mathrm{Y}$ & $\cdots$ & $\cdots$ \\
\hline MT91 646 & $\mathrm{~B} 1.5 \mathrm{~V}$ & $\mathrm{Y}$ & $\cdots$ & $\ldots$ \\
\hline МТ91 759 & B1 V & $\mathrm{Y}$ & $\ldots$ & $\ldots$ \\
\hline \multicolumn{5}{|c|}{ WR-stars } \\
\hline WR 145 & $\begin{array}{l}\text { WN7o/CE } \\
+\mathrm{O} 7 \mathrm{~V}((\mathrm{f}))\end{array}$ & $\cdots$ & $\mathrm{Y}$ & 10969 \\
\hline
\end{tabular}

Note. The last column yields the ObsID of the Chandra pointing (if any) at which intra-pointing variability is detected.

densities, which are affected by parameter correlations. Yet, we caution that because of the larger error bars on the fluxes compared to the count rates, some objects found to be variable in the previous section are found not to be significantly variable in terms of their fluxes.

\subsubsection{Known Binary Systems}

In colliding wind binary systems, one can expect phaselocked variability of the X-ray spectrum as a result of a changing column density along the line of sight toward the wind-wind interaction zone as the stars move around their common center of mass. In eccentric systems, additional variations are expected as the physical properties of the windwind interaction change with the changing separation. If the wind interaction zone is in the adiabatic regime (Stevens et al. 1992), one expects to observe an orbital modulation of the Xray flux as $1 / d$, where $d$ is the instantaneous separation between the stars, as is actually observed in the long-period system Cyg OB2 \#9 (Nazé et al. 2012b).

\subsubsection{Cyg OB2 \#3}

According to Kiminki et al. (2008), Cyg OB2 \#3 is an O6 IV +O9 III eclipsing binary system with an orbital period of 

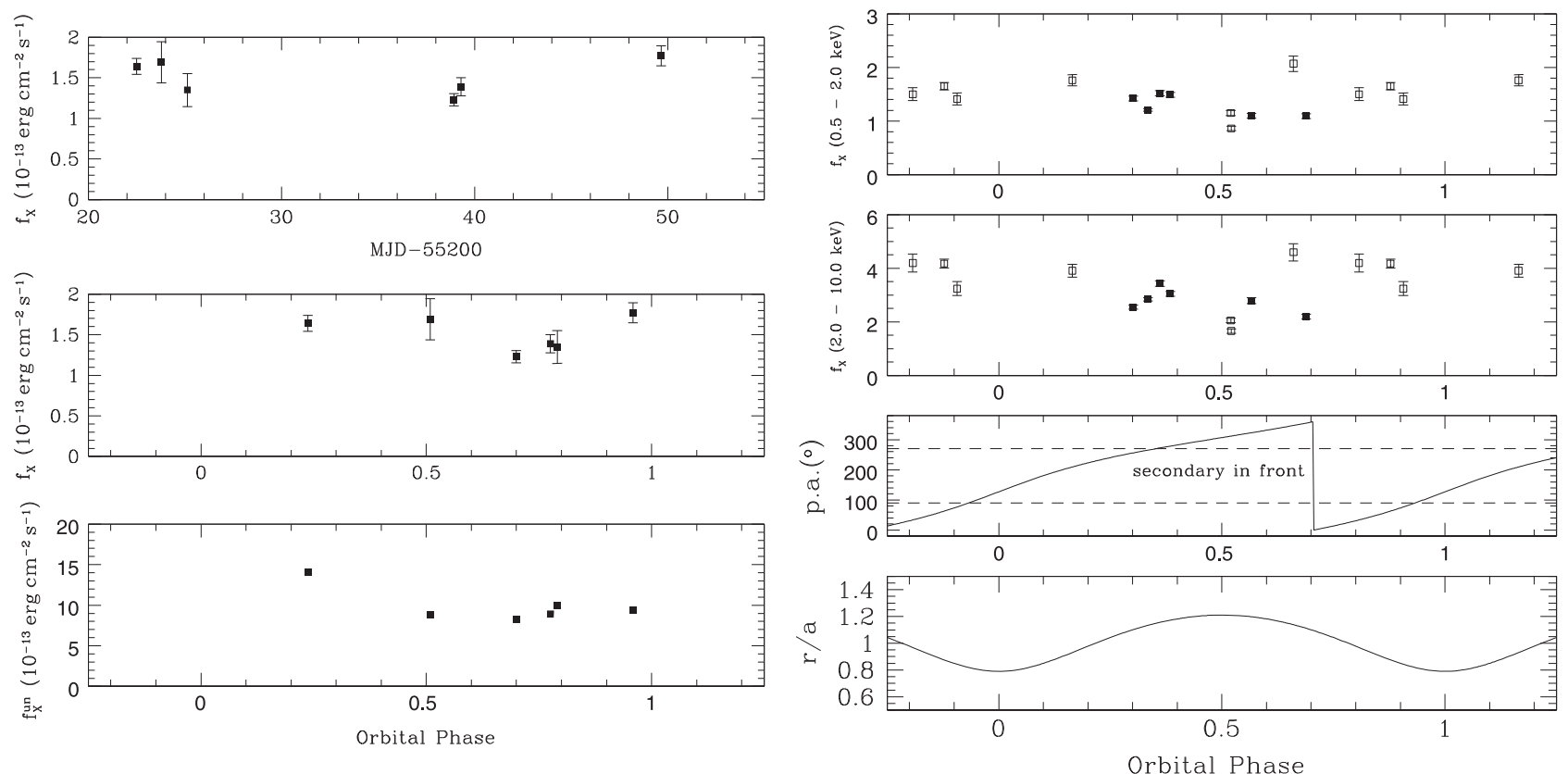

Figure 11. Left: epoch and phase-dependence of the observed and absorption-corrected X-ray fluxes of Cyg OB2 \#3. The orbital phases were computed using the ephemerides from Kiminki et al. (2008). Right, from top to bottom: observed X-ray flux in the soft $(0.5-2 \mathrm{keV})$ and hard $(2-10 \mathrm{keV})$ bands (in units $\left.10^{-13} \mathrm{erg} \mathrm{cm}^{-2} \mathrm{~s}^{-1}\right)$, position angle (defined as p.a. $=0^{\circ}$ at conjunction with the primary in front), and relative orbital separation of CPR2002 A11. The filled and open symbols stand, respectively, for the ACIS and EPIC data.

4.7464 days and an almost circular orbit $(e=0.07)$. The ACIS spectra from individual observations can be fitted using a single temperature plasma model. The intrinsic X-ray spectrum appears rather soft, with a mean ${ }^{19} k T$ of $(0.83 \pm 0.11) \mathrm{keV}$ and moderate absorption by wind material $\left(N_{\text {wind }}=(0.20 \pm 0.10) \times 10^{22} \mathrm{~cm}^{-2}\right)$.

Our observations of this star (collected over a total time span of 27 days) sample more than half of the orbital cycle (see Figure 11). The observed flux varies by $14 \%$ (standard deviation about the mean). ${ }^{20}$ This is compatible with the estimated relative errors on the fluxes of individual pointings, which are between 6 and 15\%. The ISM corrected fluxes show larger variability $(22 \%)$, but are also subject to larger uncertainties (partly due to the degeneracy between plasma temperature and wind column). In summary, we conclude that there is no clear evidence for significant orbital modulation of the X-ray flux of Cyg OB2 \#3.

\subsubsection{CPR2002 A11 = MT91 267}

The SB1 status of this O7 I star was recently reported by Kobulnicky et al. (2012) who derived an orbital period of 15.511 days and an eccentricity of 0.21. From XMM-Newton observations, its X-ray emission was found to be variable with flux variations by more than a factor two (Rauw 2011). Most individual ACIS spectra require a two temperature plasma to achieve a decent fit and for consistency, we have repeated the fitting of the EPIC data with the same model as for the ACIS spectra.

We have computed the orbital phases of the observations using the ephemerides of Kobulnicky et al. (2012). Combining

\footnotetext{
19 The uncertainties quoted correspond to the dispersion about the mean.

20 Pile-up should not be a critical issue for this source, as we estimate corrections on the observed X-ray fluxes of at most $3 \%$.
}

the XMM-Newton and Chandra data, we have an almost complete coverage of the orbital cycle, except near phase 0.0 (periastron). To better constrain the origin of the variability, we distinguish the observed fluxes over two energy domains: a soft band $(0.5-2 \mathrm{keV})$ and a hard band $(2-10 \mathrm{keV})$. The results are shown in Figure 11, along with a plot of the relative orbital separation and the position angle (defined as p.a. $=0^{\circ}$ when the $\mathrm{O} 7 \mathrm{I}$ primary star is in front). Although there are some hints that the flux in the hard band is lower near phase 0.5 , this needs confirmation. Indeed, the EPIC data show a larger amplitude of variability than the ACIS data, and observations taken at similar orbital phases sometimes display rather different fluxes. While there could be some remaining discrepancies between the calibration of the EPIC and ACIS responses (see Section 3), it seems unlikely that they could account for the observed differences. The ACIS spectra could suffer from pile-up, but based on the EPIC spectra, we have estimated that the ACIS pile-up fraction should be less than $9 \%$. Including the pileup command in the fit, leads to slightly different fluxes (typical differences of $7 \%$ ), but does not change the general appearance of the plot in Figure 11.

\subsubsection{Cyg $O B 2 \# 22$}

Cyg OB2 \#22 is a multiple system consisting of an O3 I component (star A) and an O6 V star (star B, Walborn et al. 2002; Mason et al. 2009) separated by about 1.5 arcsec. The O6 V component is itself a double system with a separation of 0.2 arcsec and a magnitude difference of 2.34 in the $z$ filter (Sota et al. 2011), and is furthermore found to be an SB1 binary with a period near 35 days (H. A. Kobulnicky et al. 2015, in preparation). Components A and B are resolved with Chandra when the star falls on-axis (ObsIDs 4511 and 10956), but are highly confused otherwise. Although the acisextract routine attempts to extract sources A and B as a pair on 


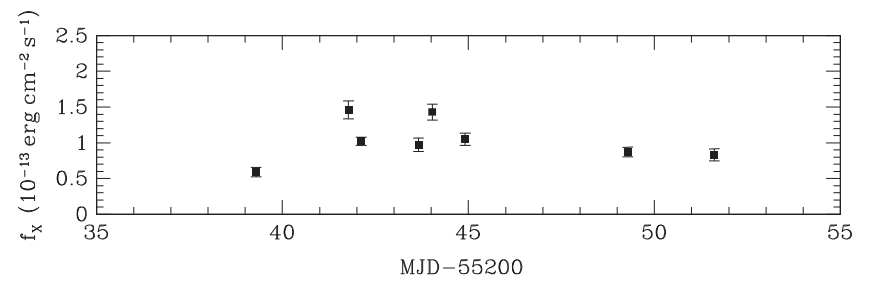

Figure 12. Combined X-ray flux of Cyg OB2 \#22 components A and B as a function of time during the ACIS survey program.

all our observations, those data taken at large off-axis angles, must be considered with caution. ${ }^{21}$

Based on six XMM-Newton observations, Rauw (2011) reported on $\mathrm{X}$-ray variability of this system with the combined X-ray flux of A + B varying by a factor 1.75 within ten days, a timescale potentially related to the newly found orbital period of Cyg OB2 \#22 B. Including all the XMM-Newton data, flux variability by a factor 2.1 was found.

To allow comparison with the EPIC data and because of the above described difficulties with the source extraction, we have summed the fluxes from the ACIS data of components A and B (see Figure 12). Although this result must be taken with caution, it seems that the ACIS data indeed support the existence of flux variations on timescales of a few days.

In principle, we should be able to combine the fluxes from the ACIS and EPIC data to perform a Fourier analysis. However, the difficulties with the source extraction described above could impact on the result. Moreover, comparing the fluxes found with XMM-Newton and Chandra, we notice that the former are systematically larger than the latter by a factor 1.5-2.0.

Therefore, while it seems that the X-ray flux of Cyg OB2 \#22 A + B is variable on a rather short term, the current data do not allow us to establish the exact value of this timescale.

\subsubsection{Cyg OB2 \#11}

Cyg OB2 \#11 (O5 I) was reported as an eccentric $(e=0.50)$ SB1 binary with a period of 72.4 days by Kobulnicky et al. (2012). According to the ephemerides provided by the latter authors, our observations span a bit more than one third of the cycle roughly centered on phase $\phi=0.4$ (periastron passage corresponding to $\phi=0.0$; see Figure 13). The observed flux varies by $17 \%$ (standard deviation about the mean), which is significant, given that the typical relative errors on individual data points are of order 5\%. Cyg OB2 \#11 is thus a good candidate for a phase-locked variation of the X-ray flux due to wind-wind interactions, and it would be interesting to collect observations near periastron passage. If the wind interaction zone is in the adiabatic regime, we would then expect the X-ray flux to be about three times larger than measured during the present campaign.

\subsubsection{MT91 771}

MT91 771 is an $07 \mathrm{~V}+\mathrm{O} 9 \mathrm{~V}$ binary with an almost circular orbit $(e=0.05 \pm 0.03)$ and a short period of 2.82 days (Kiminki et al. 2012). Our observations reveal little evidence for variability. The standard deviation around the mean

\footnotetext{
${ }^{21}$ In the X-ray to bolometric luminosity relations, we have considered the sum of components $\mathrm{A}$ and $\mathrm{B}$.
}

observed flux amounts to $9 \%$ (with relative errors on individual fluxes of $6 \%$ ).

\subsubsection{Binary Candidates}

MT91 138 and Cyg OB2 \#8c are radial velocity variable stars listed, respectively, as SB1 and SB1? by Kiminki et al. (2007), although no orbital solution is available for any of these stars.

Our data include four observations of MT91 138 over 26 days. The observed X-ray flux of this star remains constant to within $6 \%$, which is well below the typical relative uncertainty of $11 \%$.

For Cyg OB2 \#8 c, we have five observations at hand, four of which are from the survey and span two days. The fluxes of the source seem to vary at the $20 \%$ level (typical uncertainties being $7 \%$ ). One must be careful though with this source, as it falls very close to the bright Cyg OB2 \#8a which could contaminate its spectrum or the background spectrum especially for observations taken at relatively large off-axis angle.

We thus conclude that there is currently no clear indication for flux variability of these sources.

\subsubsection{Probably Single Stars}

CPR2002 A20 was observed twice, separated by 2.5 days. No significant variability is found in the observed fluxes of this source.

MT91534 was observed five times, at first during the original Cyg OB2 Chandra observation and 6 yr later in the course of the survey. Typical errors on observed fluxes of individual pointings range between 5 and $15 \%$. Except for one pointing (ObsID 10960), the fluxes are relatively constant: the standard deviation about the mean $\left(2.6 \times 10^{-14} \mathrm{erg} \mathrm{cm}^{-2} \mathrm{~s}^{-1}\right)$ of the observed fluxes amounts to $6 \%$ of the mean flux. ${ }^{22}$ ObsID 10960 is a clear outlier: the observed flux $\left(3.19 \times 10^{-13}\right.$ $\left.\operatorname{erg~cm} \mathrm{cm}^{-2} \mathrm{~s}^{-1}\right)$ is a factor 12 larger during this observation than during any other pointing and the star shows clear intrapointing variability (see Table 1). Furthermore, the plasma temperature is much higher than on average (5.0 versus $1.7 \mathrm{keV})$. These properties are reminiscent of flares in lowmass pre-main sequence stars. This flare could potentially reveal an otherwise undetectable low-mass companion near the O-star. In this context, we note that the ISM-corrected flux at ObsID $10960\left(5.14 \times 10^{-13} \mathrm{erg} \mathrm{cm}^{-2} \mathrm{~s}^{-1}\right)$ corresponds to an $\mathrm{X}$-ray luminosity of $1.2 \times 10^{32} \mathrm{erg} \mathrm{s}^{-1}$. Such values are certainly not unusual for flaring late-type pre-main-sequence stars (e.g., Wolk et al. 2005).

Cyg OB2 \#7 was observed five times with Chandra (during the original Cyg OB2 observation and 6 years later in the course of the survey, four times within 2 days), as well as at seven epochs with XMM-Newton. Based on six of the seven XMM-Newton observations, Rauw (2011) concluded that this star was constant to within $10 \%$ in the XMM-Newton data. The seventh XMM-Newton observation yields a somewhat lower flux which deviates by $22 \%$ from the mean of the previous six spectra. For the ACIS spectra, we find a dispersion about the mean of $7 \%$, supporting the idea that the source is constant at least on relatively short timescales. The EPIC data were well fitted using a single plasma component. The ACIS spectra are usually better fitted by including a second plasma

\footnotetext{
22 This star was slightly fainter than this mean level at the time of the XMMNewton observations (see its position in Figure 5).
} 

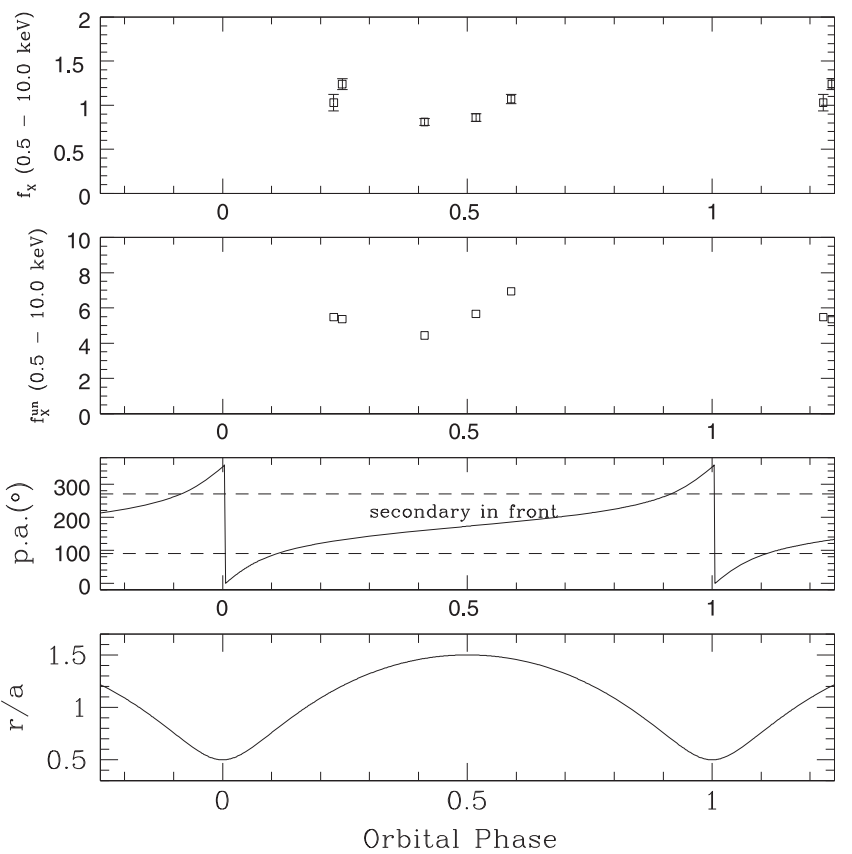

Figure 13. From top to bottom: observed and ISM-absorption corrected X-ray fluxes (in units $10^{-13} \mathrm{erg} \mathrm{cm}^{-2} \mathrm{~s}^{-1}$ ), position angle and relative orbital separation of Cyg OB2 \#11.

component, although there is a degeneracy between the plasma temperature and the wind column density, and the second (higher) plasma temperature is usually only very poorly constrained. Comparing the single plasma component fits, we find an observed flux of $(1.70 \pm 0.18) \times 10^{-13} \mathrm{erg} \mathrm{cm}^{-2} \mathrm{~s}^{-1}$ for the XMM-Newton data, while the ACIS spectra yield $(1.15 \pm 0.09) \times 10^{-13} \mathrm{erg} \mathrm{cm}^{-2} \mathrm{~s}^{-1}$, i.e., a difference of $50 \%$. A priori, pile-up should not be an issue for this object (estimated pile-up fractions are 2-4\%). We have nevertheless also performed a 2-T fit of the ACIS spectra including the pileup command. However, the observed flux remains at a low level of $(1.22 \pm 0.10) \times 10^{-13} \mathrm{erg} \mathrm{cm}^{-2} \mathrm{~s}^{-1}$. Contamination of the EPIC data by nearby weak point sources is the most likely explanation of the difference between the ACIS and EPIC fluxes.

Cyg OB2 \#8b was observed at the same five epochs as Cyg OB2 \#7. Typical errors on the determination of the observed fluxes are between 6 and $16 \%$. The standard deviation about the mean of the observed flux is $24 \%$. One has to bear in mind that this source is located in a complex region (see our remarks on Cyg OB2 \#8c).

MT91 516 was also observed five times. As this source is at the limit of a moderate pile-up, we have used the pileup command in the fits. The resulting observed fluxes are found to vary by $36 \%$ (peak to peak). This is slightly larger than the $22 \%$ flux variability found by Rauw (2011) in the XMMNewton data of this object. ${ }^{23}$ On average, the ACIS fluxes are somewhat lower $(12 \%)$ than the EPIC results, although, in the case of cyclic variability, this could also be due to a difference in the sampling of the variability cycle. Variability could hint at binarity with a likely timescale of the order of a few weeks (see Figure 14), although Kiminki et al. (2007) reported a

\footnotetext{
23 The seventh XMM-Newton observation (Nazé et al. 2012b) yields an observed flux of $5.07 \times 10^{-13} \mathrm{erg} \mathrm{cm}^{-2} \mathrm{~s}^{-1}$, slightly above the highest value of the other six observations discussed by Rauw (2011).
}

probability of $11.5 \%$ that the radial velocity variations of this star be spurious.

MT91 $213(\mathrm{~B} 0 \mathrm{~V})$ is the only B-star ${ }^{24}$ that was found to be variable and has a sufficient number of counts nearly each time it was observed to perform a spectral fit. The variations of the flux during the survey are shown in Figure 15. There are very rapid variations (by a factor 3) between ObsIDs 10944 and 10945 , i.e., within less than $8 \mathrm{hr}$. One may wonder whether the flux of ObsID 10945 is reliable, as it is the only data point that strongly deviates. However, an older observation (ObsID 4501) actually indicates a very similar, even somewhat lower, flux of $1.21 \times 10^{-14} \mathrm{erg} \mathrm{cm}^{-2} \mathrm{~s}^{-1}$. Thus, we conclude that the flux of MT91 213 indeed varies by at least a factor three and that these variations can occur on short timescales. Flares from an unseen low-mass companion are unlikely to explain this behavior, as the star seems to spend more time at a roughly constant high flux level, unlike what is seen in flaring stars.

\section{SUMMARY AND CONCLUSIONS}

In this paper, we have analyzed one of the richest samples of $\mathrm{X}$-ray data of OB stars belonging to a single association. These data have shed new light on the X-ray properties of massive stars.

We have shown that O-stars in Cyg OB2 follow a welldefined scaling relation between their X-ray and bolometric luminosities with $\log \frac{L_{\mathrm{X}}}{L_{\mathrm{bol}}}=-7.2 \pm 0.2$. This relation is in excellent agreement with the one previously derived from Chandra observations of the Carina Nebula, suggesting that any environmental effect on this relation should be quite small. Our investigation indicates that, owing to its narrow PSF, Chandra is the best mission to evaluate the X-ray emission of moderately bright and faint massive stars in crowded environments, while XMM-Newton is better suited to study the massive stars at the X-ray brighter end.

Except for the brightest O-star binaries, that we have not studied here, we do not find a general X-ray overluminosity due to colliding winds in O-star binaries, neither do we find a clear phase-locked variability in most of them. For O-star binary systems with short orbital periods, there is some tentative trend for an increase of the X-ray overluminosity with wind kinetic power, although this result clearly calls for confirmation.

B-type stars do not show a clear relationship between their $\mathrm{X}$-ray and bolometric luminosity, suggesting that their X-ray emission might come, at least for some of them, from a lowmass companion.

Finally, out of the three WC stars in Cyg OB2, probably only one (WR 144) is itself responsible for the observed level of $\mathrm{X}$-ray emission, $\log \frac{L_{\mathrm{X}}}{L_{\mathrm{bol}}}=-8.8 \pm 0.2$. The X-ray emission of the other two Wolf-Rayet stars in Cyg OB2 can be accounted for by the emission of their O-type companion as well as a moderate contribution from a wind-wind interaction zone.

The Liège team acknowledges support from Belspo through an XMM PRODEX contract, from the FRS/FNRS and from an ARC grant for Concerted Research Actions, financed by the

\footnotetext{
24 This is actually a Be star with variable emission lines (H. A. Kobulnicky et al. 2015, in preparation).
} 


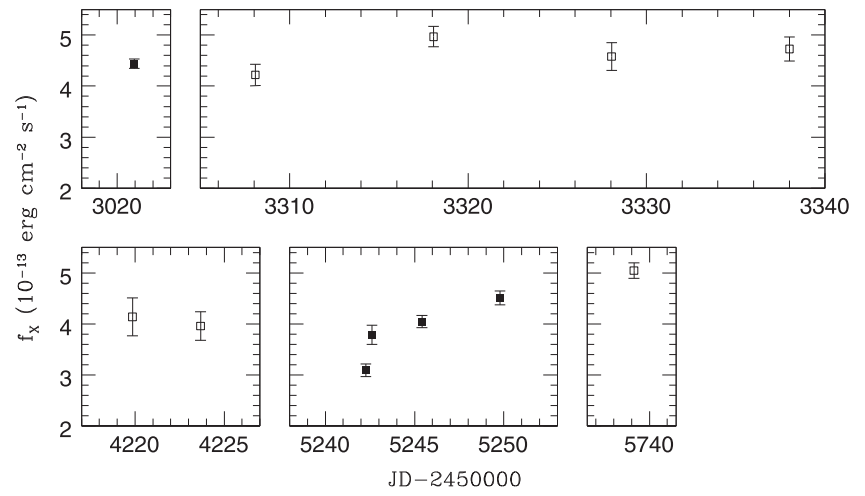

Figure 14. Observed flux of MT91516 as a function of time. The open symbols stand for XMM-Newton data from Rauw (2011) while the filled symbols indicate fluxes inferred from the ACIS data presented here.

Federation Wallonia-Brussels. N.J.W. was supported by a Royal Astronomical Society research fellowship.

Facilities: CXO, XMM.

\section{APPENDIX A}

\section{MORE DETAILS ON THE $L_{\mathrm{X}} / L_{\text {bol }}$ RELATION}

Albacete Colombo et al. (2008) argued that at least for evolved OB stars (luminosity classes I-III) in Cyg OB2, a lower scatter is achieved for power-law relations instead of simple scaling laws.

We have therefore tested a power law relation on our full data set, where $L_{\mathrm{X}}$ is allowed to scale with some power of $L_{\mathrm{bol}}$. The best-fit power-law relation (for equal weights of all 40 data points) becomes

$$
\log f_{\mathrm{X}}=(1.24 \pm 0.12) \log f_{\text {bol }}-(5.85 \pm 0.69) .
$$

Adopting instead a weighting according to the square root of the number of counts in the spectrum yields

$$
\log f_{\mathrm{X}}=(1.20 \pm 0.09) \log f_{\text {bol }}-(6.02 \pm 0.48) .
$$

In the same way, weighting the data according to the estimated errors on the fluxes leads to

$$
\log f_{\mathrm{X}}=(1.19 \pm 0.08) \log f_{\text {bol }}-(6.14 \pm 0.46) .
$$

We have then considered the 13 O-type stars of our sample with luminosity class I-III. These objects have

$$
\log L_{\mathrm{X}} / L_{\text {bol }}=-7.18 \pm 0.19
$$

(unweighted), and

$$
\log L_{\mathrm{X}} / L_{\mathrm{bol}}=-7.15 \pm 0.19
$$

(weighted; same relation for both types of weights). A powerlaw relation for the unweighted and weighted samples of giants and supergiants yields, respectively,

$$
\log f_{\mathrm{X}}=(1.04 \pm 0.09) \log f_{\text {bol }}-(6.97 \pm 0.51)
$$

and

$$
\log f_{\mathrm{X}}=(1.16 \pm 0.13) \log f_{\mathrm{bol}}-(6.33 \pm 0.65)
$$

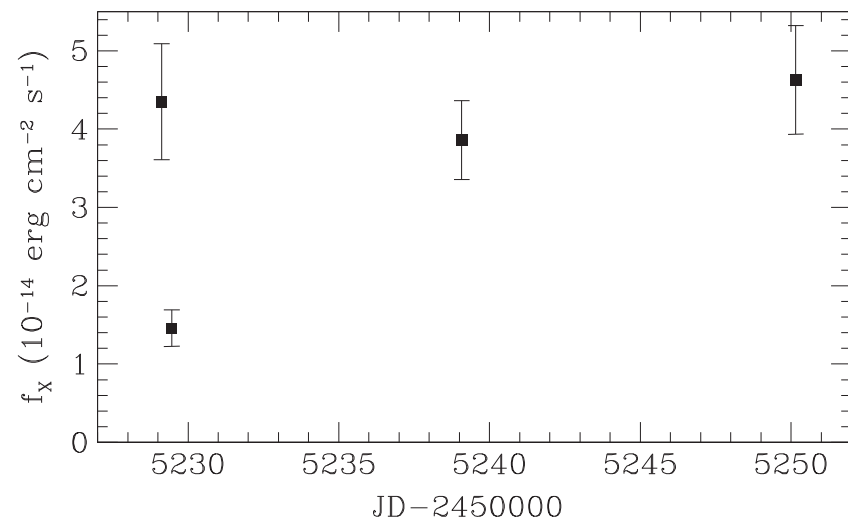

Figure 15. Observed flux of MT91 213 as a function of time during the survey.

for weighting according to the square root of the total number of counts, and

$$
\log f_{\mathrm{X}}=(1.14 \pm 0.12) \log f_{\mathrm{bol}}-(6.41 \pm 0.62)
$$

for weighting according to the estimated errors on ISMcorrected X-ray fluxes.

We see that, independently of the weighting, and of whether or not we restrict ourselves to the giants and supergiants, the exponent of $L_{\mathrm{bol}}$ deviates by less than $2 \sigma$ from unity (i.e., from a simple scaling law). Our data thus do not support the need of a power-law relation, suggesting that a scaling law is sufficient to describe the dependence of $L_{\mathrm{X}}$ on $L_{\mathrm{bol}}$ for O-stars of all luminosity classes.

Restricting ourselves to the 23 presumably single stars of the cleaned sample, we obtain the scaling relation

$$
\begin{aligned}
& \log L_{\mathrm{X}} / L_{\text {bol }}=-7.27 \pm 0.21 \\
& \log L_{\mathrm{X}} / L_{\text {bol }}=-7.22 \pm 0.21
\end{aligned}
$$

and

$$
\log L_{\mathrm{X}} / L_{\mathrm{bol}}=-7.23 \pm 0.19
$$

for the unweighted data, the data weighted according to the number of counts, and the data weighted according to the estimated errors, respectively. The corresponding power-law relations are

$$
\begin{aligned}
& \log f_{\mathrm{X}}=(1.04 \pm 0.20) \log f_{\text {bol }}-(7.06 \pm 1.16) \\
& \log f_{\mathrm{X}}=(1.13 \pm 0.27) \log f_{\text {bol }}-(6.49 \pm 1.60)
\end{aligned}
$$

and

$$
\log f_{\mathrm{X}}=(1.09 \pm 0.27) \log f_{\mathrm{bol}}-(6.72 \pm 1.58) .
$$

Within the error bars, there is no significant difference between the relations obtained from the full sample and those for presumably single stars only, even though including binaries leads to systematically higher average $\log L_{\mathrm{X}} / L_{\text {bol }}$ values (as was also found, e.g., in the Carina Nebula, Nazé et al. 2011).

\section{APPENDIX B} SPECTRAL FITS

The results of our spectral fits are summarized in Tables B1, $\mathrm{B} 2$, and $\mathrm{B} 3$. 
Table B1

Spectral Fits of O-type Stars

\begin{tabular}{|c|c|c|c|c|c|c|c|c|c|c|c|c|c|c|}
\hline Star & Spectral Type & $\begin{array}{c}\alpha \\
(\mathbf{J} 2000.0)\end{array}$ & $\begin{array}{c}\delta \\
(\mathrm{J} 2000.0)\end{array}$ & Total Counts & $\begin{array}{c}N_{\mathrm{H}}^{\mathrm{ISM}} \\
\left(10^{22} \mathrm{~cm}^{-2}\right)\end{array}$ & $\begin{array}{c}N_{\text {wind }} \\
\left(10^{22} \mathrm{~cm}^{-2}\right)\end{array}$ & $\begin{array}{c}k T_{1} \\
(\mathrm{keV})\end{array}$ & norm $_{1}$ & $\begin{array}{c}\mathrm{kT}_{2} \\
(\mathrm{keV})\end{array}$ & norm $_{2}$ & $\begin{array}{r}f_{\mathrm{X}}^{\mathrm{obs}} \\
\left(10^{-}\right. \\
\mathrm{m}\end{array}$ & $\begin{array}{l}f_{\mathrm{X}} \\
\mathrm{erg} \mathrm{c}- \\
\left.\mathrm{s}^{-1}\right)\end{array}$ & $\overline{\chi_{\nu}^{2}}$ & $\nu$ \\
\hline MT91 5 & O6 V $((f))$ & 203039.82 & +413650.5 & 15.1 & 1.11 & $0.12_{-0.12}^{+2.26}$ & $0.37_{-.31}^{+.66}$ & $8.0 \times 10^{-5}$ & $\cdots$ & $\ldots$ & 0.05 & 1.04 & 0.49 & 3 \\
\hline CPR2002 A26 & $09.5 \mathrm{~V}$ & 203057.61 & +410956.6 & 13.0 & 1.26 & 0.0 & $0.14_{-.05}^{+. .14}$ & $5.2 \times 10^{-3}$ & $\ldots$ & $\ldots$ & 0.04 & 32.5 & 0.05 & 1 \\
\hline Cyg OB2 \#1 & $\mathrm{O} 8 \mathrm{~V}$ & 203110.53 & +413153.4 & 87.0 & 0.99 & $0.16_{-.16}^{+.31}$ & $0.49_{-.19}^{+.19}$ & $8.0 \times 10^{-5}$ & $\ldots$ & $\ldots$ & 0.10 & 1.16 & 0.53 & 14 \\
\hline MT91 70 & $\mathrm{O} 9 \mathrm{~V}$ & 203118.33 & +412121.8 & 29.0 & 1.37 & $0.52_{-.52}^{+2.75}$ & $0.16_{-.11}^{+.28}$ & $2.14 \times 10^{-3}$ & $\ldots$ & $\ldots$ & 0.02 & 0.73 & 0.79 & 8 \\
\hline CPR2002 A15 & O7 I & 203136.91 & +405909.4 & 111.6 & 1.46 & 0.0 & $0.60_{-.14}^{+.13}$ & $9.9 \times 10^{-5}$ & $\ldots$ & $\ldots$ & 0.13 & 2.93 & 1.06 & 20 \\
\hline Cyg OB2 \#3 & O6 IV+O9 III & 203137.50 & +411321.1 & 1445.2 & 1.11 & $0.51_{-.17}^{+.17}$ & $0.24_{-.06}^{+.14}$ & $5.55 \times 10^{-3}$ & $0.92_{-.10}^{+.12}$ & $4.56 \times 10^{-4}$ & 1.52 & 10.83 & 1.09 & 125 \\
\hline MT91 138 & O8.5 I & 203145.40 & +411826.9 & 353.2 & 1.31 & 0.0 & $0.59_{-.11}^{+.09}$ & $1.51 \times 10^{-4}$ & $\ldots$ & $\ldots$ & 0.22 & 4.45 & 1.06 & 53 \\
\hline MT91 140 & O9.5 I & 203145.97 & +411727.0 & 92.7 & 0.31 & 0.0 & $0.28_{-.10}^{+.08}$ & $1.9 \times 10^{-5}$ & $\ldots$ & $\ldots$ & 0.08 & 0.35 & 0.62 & 16 \\
\hline Cyg OB2 \#20 & O9 III & 203149.66 & +412826.3 & 56.9 & 0.79 & 0.0 & $0.27_{-.14}^{+.19}$ & $3.6 \times 10^{-5}$ & $\ldots$ & $\ldots$ & 0.02 & 0.40 & 0.71 & 12 \\
\hline CP2012 E45 & O7 V & 203159.63 & +411450.2 & 87.6 & 1.10 & 0.0 & $0.91_{-.37}^{+.19}$ & $2.23 \times 10^{-5}$ & $\ldots$ & $\ldots$ & 0.08 & 0.64 & 0.54 & 14 \\
\hline Cyg OB2 \#4 & O7 III & 203213.84 & +412711.4 & 296.9 & 0.84 & $0.26_{-.18}^{+.20}$ & $0.37_{-.10}^{+.14}$ & $2.1 \times 10^{-4}$ & $\ldots$ & $\ldots$ & 0.16 & 1.51 & 0.74 & 42 \\
\hline Cyg OB2 \#14 & $09 \mathrm{~V}$ & 203216.57 & +412535.7 & 104.5 & 0.88 & 0.0 & $0.60_{-.42}^{+.19}$ & $1.3 \times 10^{-5}$ & $\ldots$ & $\ldots$ & 0.04 & 0.38 & 0.83 & 23 \\
\hline Cyg OB2 \#15 & O8 V & 203227.66 & +412622.1 & 41.1 & 0.85 & $0.10_{-.10}^{+.36}$ & $0.54_{-.37}^{+.92}$ & $4.3 \times 10^{-5}$ & $\ldots$ & $\ldots$ & 0.09 & 0.85 & 0.68 & 4 \\
\hline CPR2002 A11 & O7.5 III & 203231.53 & +411408.1 & 7482.8 & 1.43 & $0.23_{-.05}^{+.05}$ & $0.84_{-.06}^{+.10}$ & $8.19 \times 10^{-4}$ & $2.31_{-.18}^{+.48}$ & $4.46 \times 10^{-4}$ & 4.20 & 17.10 & 1.11 & 301 \\
\hline CPR2002 A38 & $\mathrm{O} 8 \mathrm{~V}$ & 203234.87 & +405617.0 & 117.4 & 1.08 & 0.0 & $0.85_{-.31}^{+.23}$ & $1.88 \times 10^{-5}$ & $\ldots$ & $\ldots$ & 0.06 & 0.56 & 0.83 & 67 \\
\hline Cyg OB2 \#16 & $\mathrm{O} 8 \mathrm{~V}$ & 203238.55 & +412513.6 & 286.8 & 0.86 & 0.0 & $0.68_{-.09}^{+.07}$ & $3.3 \times 10^{-5}$ & $\ldots$ & $\ldots$ & 0.11 & 1.01 & 1.29 & 48 \\
\hline Cyg OB2 \#6 & $\mathrm{O} 8 \mathrm{~V}$ & 203245.44 & +412537.6 & 264.3 & 0.88 & 0.0 & $0.49_{-.09}^{+.14}$ & $6.02 \times 10^{-5}$ & $\ldots$ & $\ldots$ & 0.12 & 1.63 & 1.09 & 43 \\
\hline Cyg OB2 \#17 & $08.5 \mathrm{~V}$ & 203250.01 & +412344.5 & 171.0 & 0.94 & 0.0 & $0.98_{-.12}^{+.14}$ & $1.77 \times 10^{-5}$ & $\ldots$ & $\ldots$ & 0.08 & 0.47 & 0.66 & 33 \\
\hline MT91 376 & O8 V & 203259.16 & +412425.3 & 96.9 & 0.94 & $0.39_{-.27}^{+.39}$ & $0.22_{-.07}^{+.07}$ & $5.67 \times 10^{-4}$ & $\ldots$ & $\ldots$ & 0.05 & 0.97 & 0.79 & 15 \\
\hline МT91 390 & $\mathrm{O} 8 \mathrm{~V}$ & 203302.92 & +411743.1 & 139.9 & 1.31 & 0.0 & $0.98_{-.19}^{+.18}$ & $2.18 \times 10^{-5}$ & $\ldots$ & $\ldots$ & 0.07 & 0.58 & 0.74 & 22 \\
\hline CPR2002 A20 & O8 II & 203302.93 & +404725.2 & 3190.7 & 1.28 & $0.76_{-.14}^{+.14}$ & $0.19_{-.04}^{+.11}$ & $4.61 \times 10^{-2}$ & $1.18_{-.06}^{+.09}$ & $2.00 \times 10^{-3}$ & 5.85 & 24.80 & 0.88 & 207 \\
\hline Cyg OB2 \#22 & $\mathrm{O} 3 \mathrm{If}+\mathrm{O} 6 \mathrm{~V}$ & 203308.77 & +411318.7 & 1522.0 & 1.35 & $0.46_{-.10}^{+.11}$ & $0.45_{-.05}^{+.05}$ & $1.58 \times 10^{-3}$ & $>2.83$ & $1.97 \times 10^{-5}$ & 1.04 & 7.85 & 1.26 & 118 \\
\hline MT91 420 & O9 V & 203309.45 & +411258.4 & 15.1 & 1.29 & 0.0 & $1.29_{-.85}^{+1.11}$ & $2.78 \times 10^{-6}$ & $\ldots$ & $\ldots$ & 0.01 & 0.05 & 0.17 & 2 \\
\hline МТ91 421 & $09.5 \mathrm{~V}$ & 203309.60 & +411300.6 & 126.1 & 1.29 & 0.0 & $0.57_{-.18}^{+.22}$ & $5.23 \times 10^{-5}$ & $\ldots$ & $\ldots$ & 0.06 & 0.80 & 0.43 & 22 \\
\hline МТ91 448 & O6 V & 203313.25 & +411328.6 & 187.7 & 1.41 & 0.0 & $0.31_{-.07}^{+.11}$ & $1.98 \times 10^{-4}$ & $\ldots$ & $\ldots$ & 0.06 & 3.82 & 1.01 & 36 \\
\hline MT91 455 & $\mathrm{O} 8 \mathrm{~V}$ & 203313.68 & +411305.7 & 173.3 & 1.21 & 0.0 & $0.70_{-.16}^{+.25}$ & $3.56 \times 10^{-5}$ & $\ldots$ & $\ldots$ & 0.07 & 0.65 & 0.81 & 29 \\
\hline Cyg OB2 \#7 & O3 If & 203314.11 & +412022.0 & 3550.2 & 1.00 & $0.81_{-.10}^{+.19}$ & $0.17_{-.03}^{+.02}$ & $3.74 \times 10^{-2}$ & $0.63_{-.05}^{+.18}$ & $8.31 \times 10^{-4}$ & 1.25 & 6.90 & 1.28 & 156 \\
\hline Cyg OB2 \#8b & O6.5 III & 203314.76 & +411841.7 & 590.5 & 0.97 & $0.40_{-.25}^{+.16}$ & $0.31_{-.05}^{+.16}$ & $5.69 \times 10^{-4}$ & $\ldots$ & $\ldots$ & 0.18 & 1.89 & 1.18 & 79 \\
\hline Cyg OB $2 \# 23$ & $09.5 \mathrm{~V}$ & 203315.77 & +412017.0 & 61.4 & 1.00 & 0.0 & $0.45_{-.17}^{+.21}$ & $1.61 \times 10^{-5}$ & $\ldots$ & $\ldots$ & 0.02 & 0.41 & 0.46 & 11 \\
\hline Cyg OB2 \#8d & $08.5 \mathrm{~V}$ & 203316.34 & +411902.0 & 149.6 & 0.99 & 0.0 & $0.61_{-.33}^{+.16}$ & $2.83 \times 10^{-5}$ & $\ldots$ & $\ldots$ & 0.07 & 0.84 & 0.93 & 30 \\
\hline Cyg OB2 \#24 & $07.5 \mathrm{~V}$ & 203317.48 & +411709.2 & 196.9 & 1.08 & 0.0 & $0.61_{-.13}^{+.13}$ & $3.24 \times 10^{-5}$ & $\ldots$ & $\ldots$ & 0.07 & 0.96 & 0.76 & 32 \\
\hline Cyg OB2 \#8c & O5 III & 203317.99 & +411831.2 & 981.5 & 0.96 & $0.43_{-.13}^{+.13}$ & $0.27_{-.08}^{+. .08}$ & $1.29 \times 10^{-3}$ & $0.93_{-.21}^{+.66}$ & $5.37 \times 10^{-5}$ & 0.40 & 3.59 & 0.90 & 95 \\
\hline MT91 485 & O8 V & 203318.02 & +412136.8 & 225.3 & 1.03 & $0.45_{-.45}^{+.50}$ & $0.12_{-.03}^{+.19}$ & $2.18 \times 10^{-2}$ & $0.98_{-.26}^{+1.13}$ & $1.61 \times 10^{-5}$ & 0.08 & 2.50 & 0.78 & 36 \\
\hline МТ91 507 & $08.5 \mathrm{~V}$ & 203321.01 & +411740.1 & 94.3 & 1.04 & 0.0 & $0.52_{-.20}^{+.32}$ & $1.63 \times 10^{-5}$ & $\ldots$ & $\ldots$ & 0.03 & 0.46 & 0.87 & 18 \\
\hline MT91 516 & $05.5 \mathrm{~V}$ & 203323.48 & +410912.6 & 7349.5 & 1.44 & $0.19_{-.08}^{+.08}$ & $0.30_{-.05}^{+.20}$ & $3.64 \times 10^{-3}$ & $1.87_{-.09}^{+.16}$ & $7.25 \times 10^{-4}$ & 4.16 & 35.10 & 1.10 & 306 \\
\hline Cyg OB2 \#25 & $08.5 \mathrm{~V}$ & 203325.53 & +413326.6 & 65.4 & 1.06 & $0.52_{-.43}^{+.72}$ & $0.19_{-.07}^{+.15}$ & $7.88 \times 10^{-4}$ & $\ldots$ & $\ldots$ & 0.03 & 0.49 & 1.34 & 15 \\
\hline MT91 534 & $07.5 \mathrm{~V}$ & 203326.74 & +411059.4 & 872.2 & 1.24 & $0.83_{-.33}^{+.37}$ & $0.16_{-.03}^{+.06}$ & $1.21 \times 10^{-2}$ & $2.25_{-.25}^{+.42}$ & $9.52 \times 10^{-5}$ & 0.55 & 1.73 & 1.12 & 123 \\
\hline Cyg OB2 \#74 & $\mathrm{O} 8 \mathrm{~V}$ & 203330.30 & +413557.9 & 127.0 & 1.26 & 0.0 & $0.60_{-.14}^{+.12}$ & $4.72 \times 10^{-5}$ & $\ldots$ & $\ldots$ & 0.08 & 1.40 & 0.77 & 24 \\
\hline MT91 611 & $\mathrm{O} 7 \mathrm{~V}$ & 203340.90 & +413017.9 & 21.9 & 1.06 & 0.0 & $0.79_{-.72}^{+.54}$ & $5.0 \times 10^{-6}$ & $\ldots$ & $\ldots$ & 0.02 & 0.15 & 1.22 & 2 \\
\hline Cyg OB2 \#10 & O9 I & 203346.11 & +413300.7 & 253.6 & 1.04 & 0.0 & $0.60_{-.09}^{+.13}$ & $5.95 \times 10^{-5}$ & $\ldots$ & $\ldots$ & 0.13 & 1.77 & 1.22 & 46 \\
\hline Cyg OB2 \#27 & $09.5 \mathrm{~V}+\mathrm{BO} \mathrm{V}$ & 203359.56 & +411735.5 & 113.3 & 1.11 & 0.0 & $0.60_{-.15}^{+.15}$ & $2.38 \times 10^{-5}$ & $\ldots$ & $\ldots$ & 0.05 & 0.70 & 0.70 & 22 \\
\hline
\end{tabular}


Table B2

Same as Table B1, but for B-type Stars

\begin{tabular}{|c|c|c|c|c|c|c|c|c|c|c|c|c|}
\hline Star & $\begin{array}{l}\text { Spectral } \\
\text { Type }\end{array}$ & $\begin{array}{c}\alpha \\
(\mathrm{J} 2000.0)\end{array}$ & $\begin{array}{c}\delta \\
(\mathrm{J} 2000.0)\end{array}$ & $\begin{array}{c}\text { Total } \\
\text { Counts }\end{array}$ & $\begin{array}{c}N_{\mathrm{H}}^{\mathrm{ISM}} \\
\left(10^{22} \mathrm{~cm}^{-2}\right)\end{array}$ & $\begin{array}{c}N_{\text {wind }} \\
\left(10^{22} \mathrm{~cm}^{-2}\right)\end{array}$ & $\begin{array}{c}k T_{1} \\
(\mathrm{keV})\end{array}$ & norm $_{1}$ & $\begin{array}{l}f_{\mathrm{X}}^{\mathrm{obs}} \\
\left(10^{-1}\right. \\
\mathrm{m}^{-2}\end{array}$ & $\begin{array}{r}f_{\mathrm{X}} \\
\text { erg c- } \\
\left.\mathrm{s}^{-1}\right)\end{array}$ & $\chi_{\nu}^{2}$ & $\nu$ \\
\hline MT91 20 & B0 V+O9 I & 203051.07 & +412021.6 & 10.7 & 1.37 & $\ldots$ & $\ldots$ & $\ldots$ & 0.16 & 0.36 & $\ldots$ & $\ldots$ \\
\hline MT91 42 & B2 V & 203059.56 & +413600.2 & 5.8 & 0.84 & $\ldots$ & $\ldots$ & $\ldots$ & 0.26 & 0.50 & $\ldots$ & $\ldots$ \\
\hline CPR 2002 A 30 & B2 V & 203122.11 & +411202.9 & 4.0 & 1.09 & $\ldots$ & $\ldots$ & $\ldots$ & 0.62 & 1.31 & $\ldots$ & $\cdots$ \\
\hline MT91 103 & $\begin{array}{l}\mathrm{B} 1 \mathrm{~V} \\
+\mathrm{B} 2 \mathrm{~V}\end{array}$ & 203133.35 & +412248.2 & 144.8 & 1.26 & 0.0 & $2.46_{-.52}^{+.86}$ & $2.10 \times 10^{-5}$ & 1.42 & 3.10 & 1.14 & 30 \\
\hline MT91 129 & B3 V & 203141.66 & +412820.3 & 49.3 & 0.86 & 0.0 & $3.0_{-1.4}^{+11.3}$ & $6.2 \times 10^{-6}$ & 0.55 & 0.96 & 0.84 & 7 \\
\hline MT91 174 & B2 III & 203156.96 & +413148.0 & 6.8 & 0.80 & $\ldots$ & $\ldots$ & $\ldots$ & 0.05 & 0.10 & $\ldots$ & $\ldots$ \\
\hline MT91 179 & B3 V & 203159.93 & +413712.8 & 4.4 & 0.79 & $\ldots$ & $\ldots$ & $\ldots$ & 0.07 & 0.14 & $\ldots$ & $\cdots$ \\
\hline MT91 213 & B0 V & 203213.13 & +412724.3 & 270.6 & 0.79 & 0.0 & $3.97_{-.96}^{+2.06}$ & $2.4 \times 10^{-5}$ & 2.62 & 4.05 & 1.11 & 48 \\
\hline MT91 216 & $\mathrm{~B} 1.5 \mathrm{~V}$ & 203213.82 & +412741.6 & 31.1 & 0.80 & 0.0 & $2.63_{-1.91}^{+4.38}$ & $3.9 \times 10^{-6}$ & 0.32 & 0.58 & 0.48 & 5 \\
\hline MT91 220 & B1 V & 203214.61 & +412233.5 & 8.0 & 1.00 & $\ldots$ & $\ldots$ & $\ldots$ & 0.08 & 0.17 & $\ldots$ & $\ldots$ \\
\hline MT91 221 & $\mathrm{~B} 2 \mathrm{~V}$ & 203214.70 & +412739.6 & 203.3 & 0.86 & $0.51_{-.50}^{+.34}$ & $1.21_{-.44}^{+.64}$ & $2.95 \times 10^{-5}$ & 1.09 & 2.40 & 1.00 & 36 \\
\hline MT91 239 & B $4 \mathrm{~V}$ & 203221.77 & +413425.4 & 7.8 & 0.74 & $\ldots$ & $\ldots$ & $\ldots$ & 0.09 & 0.16 & $\ldots$ & $\ldots$ \\
\hline MT91 250 & B2 III & 203226.10 & +412940.9 & 7.4 & 0.72 & $\ldots$ & $\ldots$ & $\ldots$ & 0.06 & 0.11 & $\ldots$ & $\ldots$ \\
\hline MT91 252 & $\begin{array}{l}\text { B1.5 III } \\
+\mathrm{B} 1 \mathrm{~V}\end{array}$ & 203226.53 & +411913.4 & 14.4 & 1.00 & $\cdots$ & $\cdots$ & $\cdots$ & 0.21 & 0.42 & $\cdots$ & ... \\
\hline MT91 255 & B2 III & 203227.25 & +412156.6 & 35.7 & 0.92 & 0.0 & $3.0_{-2.0}^{+11.4}$ & $4.8 \times 10^{-6}$ & 0.41 & 0.74 & 0.47 & 9 \\
\hline Cyg OB2 \#21 & $\mathrm{B} 0.5 \mathrm{~V}$ & 203227.77 & +412852.1 & 46.2 & 0.71 & 0.0 & $0.61_{-.35}^{+.26}$ & $6.0 \times 10^{-6}$ & 0.23 & 1.79 & 0.74 & 8 \\
\hline MT91 271 & B4 V & 203232.42 & +412257.9 & 7.1 & 0.95 & $\ldots$ & $\ldots$ & $\ldots$ & $\ldots$ & $\ldots$ & $\ldots$ & $\ldots$ \\
\hline MT91 295 & $\mathrm{~B} 2 \mathrm{~V}$ & 203237.72 & +412615.5 & 43.3 & 0.81 & 0.0 & $4.4_{-2.2}^{+\ldots}$ & $3.8 \times 10^{-6}$ & 0.44 & 0.67 & 0.65 & 10 \\
\hline MT91 298 & B3 V & 203238.35 & +412857.0 & 43.7 & 0.81 & $0.21_{-.21}^{+1.07}$ & $1.60_{-.72}^{+1.09}$ & $6.7 \times 10^{-6}$ & 0.37 & 0.75 & 0.59 & 7 \\
\hline MT91 300 & $\mathrm{~B} 1 \mathrm{~V}$ & 203238.89 & +412520.3 & 22.1 & 0.82 & 0.0 & $4.35_{-3.90}^{+\ldots}$ & $2.8 \times 10^{-6}$ & 0.31 & 0.48 & 0.67 & 3 \\
\hline CPR 2002 A31 & $\mathrm{B} 0.5 \mathrm{~V}$ & 203239.50 & +405247.5 & 18.6 & 1.29 & 0.0 & $0.27_{-.27}^{+.99}$ & $4.0 \times 10^{-5}$ & 0.09 & 7.19 & 1.31 & 21 \\
\hline MT91 311 & $\begin{array}{l}\mathrm{B} 2 \mathrm{~V} \\
+\mathrm{B} 3 \mathrm{~V}\end{array}$ & 203242.88 & +412016.4 & 194.2 & 0.90 & $0.39_{-.26}^{+.23}$ & $1.10_{-.16}^{+.23}$ & $2.1 \times 10^{-5}$ & 0.74 & 2.10 & 1.15 & 36 \\
\hline MT91 322 & $\mathrm{~B} 2.5 \mathrm{~V}$ & 203246.47 & +412422.0 & 93.6 & 0.87 & $0.55_{-.39}^{+.35}$ & $1.15_{-.23}^{+.48}$ & $2.36 \times 10^{-5}$ & 0.80 & 1.83 & 0.67 & 17 \\
\hline MT91 336 & B3 III & 203249.65 & +412536.4 & 97.6 & 0.77 & 0.0 & $2.39_{-.86}^{+1.16}$ & $1.37 \times 10^{-5}$ & 1.07 & 2.01 & 0.83 & 17 \\
\hline Cyg OB2 \#37 & B3 V & 203254.40 & +411521.9 & 14.5 & 1.33 & $\ldots$ & $\ldots$ & $\ldots$ & 0.10 & 0.23 & $\ldots$ & $\ldots$ \\
\hline MT91 372 & $\begin{array}{l}\text { B0 V } \\
+\mathrm{B} 2 \mathrm{~V}\end{array}$ & 203258.90 & +410430.1 & 72.4 & 1.37 & 0.0 & $1.97_{-.82}^{+1.91}$ & $1.68 \times 10^{-5}$ & 0.91 & 2.47 & 0.98 & 13 \\
\hline MT91 378 & $\mathrm{~B} 0 \mathrm{~V}$ & 203259.63 & +411514.7 & 61.9 & 1.33 & 1.44 & $0.18_{-.13}^{+.12}$ & $2.43 \times 10^{-3}$ & 0.13 & 0.79 & 0.93 & 15 \\
\hline MT91 400 & B1 V & 203305.16 & +411751.2 & 7.4 & 1.05 & $\ldots$ & $\ldots$ & $\ldots$ & 0.04 & 0.09 & $\ldots$ & $\ldots$ \\
\hline MT91 425 & B $0 \mathrm{~V}$ & 203310.10 & +411310.2 & 17.0 & 1.24 & $2.20_{-2.20}^{+6.95}$ & $0.07_{-.04}^{+2.08}$ & 29.7 & 0.03 & 0.41 & 0.32 & 3 \\
\hline MT91 428 & $\mathrm{~B} 1 \mathrm{~V}$ & 203310.47 & +412057.4 & 32.8 & 1.14 & 0.0 & $3.6_{-2.8}^{+\ldots}$ & $3.3 \times 10^{-6}$ & 0.31 & 0.54 & 1.06 & 6 \\
\hline MT91 429 & $\begin{array}{l}\text { B0 V } \\
+\mathrm{B} 3 \mathrm{~V}\end{array}$ & 203310.57 & +412222.7 & 11.7 & 1.02 & $\cdots$ & $\ldots$ & $\ldots$ & 0.08 & 0.13 & $\cdots$ & ... \\
\hline MT91 435 & B0 V & 203311.09 & +411032.3 & 14.2 & 1.38 & $0.11_{-0.10}^{+3.68}$ & $0.42_{-.32}^{+0.97}$ & $9.0 \times 10^{-6}$ & 0.05 & 1.37 & 0.42 & 3 \\
\hline MT91 453 & B5 V & 203313.34 & +412639.4 & 15.5 & 0.73 & 0.0 & $2.32_{-1.95}^{+\ldots}$ & $2.1 \times 10^{-6}$ & 0.17 & 0.31 & 0.59 & 2 \\
\hline MT91 459 & B5 V & 203314.33 & +411933.0 & 104.5 & 1.10 & $0.42_{-.42}^{+.46}$ & $0.30_{-.11}^{+.26}$ & $1.26 \times 10^{-4}$ & 0.28 & 3.69 & 1.11 & 20 \\
\hline MT91 467 & $\mathrm{~B} 1 \mathrm{~V}$ & 203315.27 & +412956.5 & 52.4 & 1.03 & 0.0 & $3.16_{-1.46}^{+4.91}$ & $9.0 \times 10^{-6}$ & 0.79 & 1.42 & 0.65 & 9 \\
\hline MT91 477 & B0 $\mathrm{V}$ & 203317.41 & +411238.7 & 17.9 & 1.23 & $1.23_{-1.23}^{+4.88}$ & $0.38_{-.32}^{+1.45}$ & $3.37 \times 10^{-5}$ & 0.06 & 0.23 & 0.65 & 3 \\
\hline MT91 509 & B0 III & 203321.02 & +413552.4 & 0.8 & 1.15 & $\ldots$ & $\ldots$ & & 0.04 & 0.10 & $\ldots$ & $\ldots$ \\
\hline Cyg OB2 \#18 & $\mathrm{B} 1 \mathrm{Ib}$ & 203330.77 & +411522.7 & 28.3 & 1.10 & 0.0 & $0.18_{-.09}^{+.69}$ & $9.1 \times 10^{-5}$ & 0.06 & 10.70 & 1.06 & 9 \\
\hline MT91 561 & B2 V & 203331.62 & +412146.7 & 6.6 & 0.86 & $\ldots$ & $\ldots$ & $\ldots$ & $\ldots$ & $\ldots$ & $\ldots$ & $\ldots$ \\
\hline MT91 573 & B3 I & 203333.97 & +411938.1 & 21.2 & 0.98 & 0.0 & $4.02_{-3.29}^{+\ldots}$ & $5.4 \times 10^{-6}$ & 0.57 & 0.92 & 0.95 & 2 \\
\hline Cyg OB2 \#70 & B0 $\mathrm{V}$ & 203337.00 & +411611.1 & 91.5 & 1.09 & $0.13_{-.13}^{+.42}$ & $0.59_{-.23}^{+.25}$ & $1.90 \times 10^{-5}$ & 0.31 & 3.47 & 0.85 & 24 \\
\hline CP2012 E52 & B0 Ib & 203338.21 & +405341.1 & 101.4 & 1.10 & 0.0 & $0.97_{-.25}^{+.21}$ & $3.44 \times 10^{-5}$ & 1.29 & 9.24 & 0.64 & 17 \\
\hline Cyg OB2 \#19 & B0 Iab & 203339.09 & +411925.9 & 159.2 & 0.97 & $0.28_{-.22}^{+.34}$ & $0.41_{-.14}^{+.14}$ & $7.52 \times 10^{-5}$ & 0.59 & 5.96 & 0.71 & 27 \\
\hline MT91 620 & B0 V & 203342.30 & +411146.4 & 31.0 & 1.17 & 0.0 & $0.81_{-.54}^{+.52}$ & $3.1 \times 10^{-6}$ & 0.09 & 0.92 & 1.71 & 6 \\
\hline MT91 621 & $\mathrm{~B} 1 \mathrm{~V}$ & 203342.55 & +411457.0 & 9.1 & 1.21 & $\ldots$ & $\ldots$ & $\ldots$ & 0.10 & 0.22 & $\ldots$ & $\ldots$ \\
\hline MT91 635 & B1 III & 203346.83 & +410801.6 & 169.1 & 1.10 & 0.0 & $2.58_{-1.00}^{+.96}$ & $1.35 \times 10^{-5}$ & 0.99 & 2.01 & 1.23 & 38 \\
\hline Cyg OB2 \#26 & B1 III & 203347.81 & +412041.2 & 73.2 & 1.01 & $0.86_{-.40}^{+.65}$ & $0.73_{-.24}^{+.31}$ & $2.41 \times 10^{-5}$ & 0.32 & 0.89 & 0.87 & 17 \\
\hline MT91 639 & $\mathrm{~B} 2 \mathrm{~V}$ & 203347.84 & +410908.2 & 30.3 & 1.12 & 0.0 & $1.1_{-.8}^{+16.1}$ & $3.1 \times 10^{-6}$ & 0.13 & 0.70 & 0.93 & 7 \\
\hline MT91 646 & $\mathrm{~B} 1.5 \mathrm{~V}$ & 203348.83 & +411940.5 & 107.9 & 0.92 & $1.42_{-.73}^{+1.37}$ & $1.64_{-.56}^{+.69}$ & $2.22 \times 10^{-5}$ & 0.77 & 1.03 & 0.88 & 22 \\
\hline MT91 692 & B0 V & 203359.23 & +410537.9 & 6.7 & 1.10 & $\ldots$ & $\ldots$ & $\ldots$ & 0.26 & 0.54 & $\ldots$ & $\ldots$ \\
\hline MT91 720 & $\begin{array}{c}\mathrm{B} 0.5 \mathrm{~V} \\
+\mathrm{B} 1.5 \mathrm{~V}\end{array}$ & 203406.02 & +410809.3 & 7.2 & 1.29 & $\cdots$ & $\cdots$ & $\ldots$ & 0.09 & 0.21 & $\cdots$ & ... \\
\hline MT91 759 & B1 V & 203424.60 & +412624.7 & 142.6 & 1.08 & 0.0 & $2.59_{-.63}^{+1.13}$ & $2.33 \times 10^{-5}$ & 1.74 & 3.48 & 0.82 & 24 \\
\hline CPR 2002 A36 & $\begin{array}{c}\text { B0 Ib } \\
+ \text { B0 III }\end{array}$ & 203458.78 & +413617.3 & 269.3 & 1.19 & 0.0 & $0.90_{-.22}^{+.10}$ & $1.25 \times 10^{-4}$ & 4.01 & 36.01 & 0.64 & 40 \\
\hline
\end{tabular}


Table B3

Same as Table B1, but for Wolf-Rayet Stars

\begin{tabular}{|c|c|c|c|c|c|c|c|c|c|c|c|c|c|c|c|}
\hline Star & Spectral Type & $\begin{array}{c}\alpha \\
(\mathrm{J} 2000.0)\end{array}$ & $\begin{array}{c}\delta \\
(\mathrm{J} 2000.0)\end{array}$ & Total Counts & $\begin{array}{c}N_{\mathrm{H}}^{\mathrm{ISM}} \\
\left(10^{22} \mathrm{~m}^{-2}\right)\end{array}$ & Abund & $\begin{array}{c}N_{\text {wind }} \\
\left(10^{22} \mathrm{~cm}^{-2}\right)\end{array}$ & $\begin{array}{c}k T_{1} \\
(\mathrm{keV})\end{array}$ & norm $_{1}$ & $\begin{array}{l}\mathrm{kT}_{2} \\
(\mathrm{keV})\end{array}$ & norm $_{2}$ & $\begin{array}{l}f_{\mathrm{X}}^{\text {obs }} \\
\left(10^{-13}\right. \\
\mathrm{m}^{-2}\end{array}$ & $\begin{array}{l}f_{\mathrm{X}} \\
\text { erg c- } \\
\mathrm{s}^{-1} \text { ) }\end{array}$ & $\overline{\chi_{\nu}^{2}}$ & $\nu$ \\
\hline WR 144 & WC4 & 203202.92 & +411518.8 & 5.8 & 0.97 & $\ldots$ & $\ldots$ & $\ldots$ & $\ldots$ & $\ldots$ & $\ldots$ & $\ldots$ & $\ldots$ & $\ldots$ & $\ldots$ \\
\hline WR 145 & $\mathrm{WN7o/CE}+\mathrm{O} 7 \mathrm{~V}((\mathrm{f}))$ & 203206.26 & +404829.6 & 696.3 & 0.97 & WC & $\left(3.4_{-.9}^{+.6}\right) \times 10^{-3}$ & $1.59_{.17}^{+.38}$ & $4.05 \times 10^{-6}$ & $\ldots$ & $\cdots$ & 5.39 & 6.56 & 1.08 & 110 \\
\hline WR 145 & $\mathrm{WN7o/CE}+\mathrm{O} 7 \mathrm{~V}((\mathrm{f}))$ & 203206.26 & +404829.6 & 696.3 & 0.97 & solar & $2.68_{-.73}^{+.53}$ & $1.60_{.19}^{+.43}$ & $2.12 \times 10^{-3}$ & $\ldots$ & $\ldots$ & 5.33 & 6.50 & 1.05 & 110 \\
\hline WR 146 & WC6+O8 III & 203547.07 & +412244.7 & 1827.5 & 1.32 & WC & $\left(6.2_{-1.7}^{+2.1}\right) \times 10^{-4}$ & $0.36_{.08}^{+.09}$ & $4.43 \times 10^{-6}$ & $2.10_{-.22}^{+.25}$ & $8.28 \times 10^{-7}$ & 2.92 & 13.02 & 1.32 & 176 \\
\hline WR 146 & WC6+O8 III & 203547.07 & +412244.7 & 1827.5 & 1.32 & solar & $0.46_{-0.17}^{+.20}$ & $0.36_{.08}^{+.11}$ & $2.88 \times 10^{-3}$ & $2.14_{-.27}^{+.40}$ & $4.06 \times 10^{-4}$ & 2.95 & 13.60 & 1.23 & 176 \\
\hline
\end{tabular}




\section{REFERENCES}

Albacete Colombo, J. F., Micela, G., Flaccomio, E., \& Sciortino, S. 2008, in ASP Conf. Ser. 388, Mass Loss from Stars and the Evolution of Stellar Clusters, ed. A. de Koter, L. Smith, \& R. Waters (San Francisco, CA: ASP), 139

Anders, E., \& Grevesse, N. 1989, GeCoA, 53, 197

Arnaud, K. A. 1996, in ASP Conf. Ser. 101, Astronomical Data Analysis Software and Systems V, ed. G. Jacoby, \& J. Barnes (San Francisco, CA: ASP), 17

Asplund, M., Grevesse, N., Sauval, A. J., \& Scott, P. 2009, ARA\&A, 47, 481

Babel, J., \& Montmerle, T. 1997, ApJL, 485, L29

Berghöfer, T. W., Schmitt, J. H. M. M., Danner, R., \& Cassinelli, J. P. 1997, A\&A, 322, 167

Blomme, R., De Becker, M., Volpi, D., \& Rauw, G. 2010, A\&A, 519, A111

Bohlin, R. C., Savage, B. D., \& Drake, J. F. 1978, ApJ, 224, 132

Broos, P. S., Townsley, L. K., Feigelson, E. D., et al. 2010, ApJ, 714, 1582

Cazorla, C., Nazé, Y., \& Rauw, G. 2014, A\&A, 561, A92

Comerón, F., Pasquali, A., Rodighiero, G., et al. 2002, A\&A, 389, 874

Davis, J. E. 2001, ApJ, 562, 575

De Becker, M., Rauw, G., Sana, H., et al. 2006, MNRAS, 371, 1280

De Becker, M. 2013, NewA, 25, 7

Dougherty, S. M., Williams, P. M., van der Hucht, K. A., Bode, M. F., \& Davis, R. J. 1996, MNRAS, 280, 963

Dougherty, S. M., Williams, P. M., \& Pollacco, D. L. 2000, MNRAS, 316, 143

Evans, N. R., DeGioia-Eastwood, K., Gagné, M., et al. 2011, ApJS, 194, 13

Feldmeier, A., Puls, J., \& Pauldrach, A. W. A. 1997, A\&A, 322, 878

Gagné, M., Fehon, G., Savoy, M. R., et al. 2011, ApJS, 194, 5

Gosset, E., Nazé, Y., Claeskens, J.-F., et al. 2005, A\&A, 492, 685

Hanson, M. M. 2003, ApJ, 597, 957

Harnden, F. ,R., Jr, Branduardi, G., Gorenstein, P., et al. 1979, ApJL, 234, L51

Hervé, A., Rauw, G., \& Nazé, Y. 2013, A\&A, 551, A83

Kiminki, D. C., Kobulnicky, H. A., Kinemuchi, K., et al. 2007, ApJ, 664, 1102

Kiminki, D. C., McSwain, M. V., \& Kobulnicky, H. A. 2008, ApJ, 679, 1478

Kiminki, D. C., Kobulnicky, H. A., Ewing, I., et al. 2012, ApJ, 747, 41

Kobulnicky, H. A., Kiminki, D. C., Lundquist, M. J., et al. 2014, ApJS, 213, 34

Kobulnicky, H. A., Smullen, R., Kiminki, D. C., et al. 2012, ApJ, 756, 50

Lamers, H. J. G. L. M., Snow, T. P., \& Lindholm, D. M. 1995, ApJ, 455, 269

Lépine, S., Wallace, D., Shara, M. M., Moffat, A. F. J., \& Niemela, V. S. 2001, AJ, 122, 3407

Linder, N., Rauw, G., Manfroid, J., et al. 2009, A\&A, 495, 231

Lundström, I., \& Stenholm, B. 1984, A\&AS, 58, 163

Martins, F., \& Plez, B. 2006, A\&A, 457, 637

Mason, B. D., Hartkopf, W. I., Gies, D. R., Henry, T. J., \& Helsel, J. W. 2009, AJ, 137, 3358

Muijres, L. E., Vink, J. S., de Koter, A., Müller, P. E., \& Langer, N. 2012, A\&A, 537, A37

Muntean, V., Moffat, A. F. J., Chené, A. N., \& de la Chevrotière, A. 2009, MNRAS, 399, 1977
Nazé, Y. 2009, A\&A, 506, 1055

Nazé, Y., Broos, P. S., Oskinova, L., et al. 2011, ApJS, 194, 7

Nazé, Y., Rauw, G., \& Hutsemékers, D. 2012, A\&A, 538, A47

Nazé, Y., Mahy, L., Damerdji, Y., et al. 2012, A\&A, 546, A37

Nazé, Y., Oskinova, L. M., \& Gosset, E. 2013, ApJ, 763, 143

Nazé, Y., Rauw, G., Sana, H., \& Corcoran, M. F. 2013, A\&A, 555, A83

Negueruela, I., Marco, A., Herrero, A., \& Clark, J. S. 2008, A\&A, 487, 575

Niemela, V. S., Shara, M. M., Wallace, D. J., Zurek, D. R., \& Moffat, A. F. J. 1998, AJ, 115, 2047

Oskinova, L. M. 2005, MNRAS, 361, 679

Oskinova, L. M., Ignace, R., Hamann, W.-R., Pollock, A. M. T., \& Brown, J. C. 2003, A\&A, 402, 755

Oskinova, L. M., Todt, H., Ignace, R., et al. 2011, MNRAS, 416, 1456

Owocki, S. P., \& Cohen, D. H. 1999, ApJ, 520, 833

Owocki, S. P., Sundqvist, J. O., Cohen, D. H., \& Gayley, K. G. 2013, MNRAS, 429, 3379

Pittard, J. M., \& Stevens, I. R. 1997, MNRAS, 292, 298

Pollock, A. M. T. 1987, ApJ, 320, 283

Pollock, A. M. T., Haberl, F., \& Corcoran, M. F. 1995, in Wolf-Rayet Stars: Binaries, Colliding Winds, Evolution, ed. K. A. van der Hucht, \& P. M. Williams (Dordrecht: Kluwer), 512

Rauw, G. 2011, A\&A, 536, 31

Rieke, G. H., \& Lebofsky, M. J. 1985, ApJ, 288, 618

Sana, H., Rauw, G., Nazé, Y., Gosset, E., \& Vreux, J.-M. 2006, MNRAS, 372,661

Sander, A., Hamann, W.-R., \& Todt, H. 2012, A\&A, 540, 144

Schellenberger, G., Reiprich, T., \& Lovisari, L. 2013, http://web.mit.edu/ iachec/meetings/2013/Presentations/Schellenberger.pdf

Sciortino, S., Vaiana, G. S., Harnden, F. R., Jr, et al. 1990, ApJ, 361, 621

Setia Gunawan, D. Y. A., de Bruyn, A. G., van der Hucht, K. A., \& Williams, P. M. 2000, A\&A, 356, 676

Smith, R. K., \& Brickhouse, N. S. 2001, ApJL, 556, L91

Sota, A., Maíz Apellániz, J., Walborn, N. R., et al. 2011, ApJS, 193, 24

Stevens, I. R., Blondin, J. M., \& Pollock, A. M. T. 1992, ApJ, 386, 265

ud-Doula, A., \& Owocki, S. P. 2002, ApJ, 576, 413

van der Hucht, K. A. 2001, NewAR, 45, 135

Vink, J. S., de Koter, A., \& Lamers, H. J. G. L. M. 2001, A\&A, 369, 574

Vink, J. S., Muijres, L. E., Anthonisse, B., et al. 2011, A\&A, 531, A132

Walborn, N. R., Howarth, I. D., Lennon, D. J., et al. 2002, AJ, 123, 2754

Wessolowski, U. 1996, Röntgenstrahlung from the Universe, Vol. 263 ed.

H. U. Zimmermann, J. Trümper, \& H. Yorke (Garching: Max-Planck Institute for Extraterrestrial Physics), 75

Wolk, S. J., Harnden, F. R., Jr, Flaccomio, E., et al. 2005, ApJS, 160, 423

Wright, N. J., \& Drake, J. J. 2009, ApJS, 184, 84

Wright, N. J., Drake, J. J., Drew, J. E., \& Vink, J. S. 2010, ApJ, 713, 871

Wright, N. J., Drake, J. J., Guarcello, M. G., et al. 2014, ApJS, in press (arXiv: 1408.6579)

Wright, N. J., Drew, J. E., \& Mohr-Smith, M. 2015, MNRAS, 449, 741

Zhekov, S. A. 2012, MNRAS, 422, 1332 